\title{
Large time asymptotics of growth models on space-like paths II: PNG and parallel TASEP
}

\author{
Alexei Borodin*, Patrik L. Ferrari \\ Tomohiro Sasamoto ${ }^{\ddagger}$
}

July 27, 2007

\begin{abstract}
We consider the polynuclear growth (PNG) model in $1+1$ dimension with flat initial condition and no extra constraints. The joint distributions of surface height at finitely many points at a fixed time moment are given as marginals of a signed determinantal point process. The long time scaling limit of the surface height is shown to coincide with the Airy 1 process. This result holds more generally for the observation points located along any space-like path in the spacetime plane. We also obtain the corresponding results for the discrete time TASEP (totally asymmetric simple exclusion process) with parallel update.
\end{abstract}

\section{Introduction}

The main focus of this work is a stochastic growth model in $1+1$ dimensions, called the polynuclear growth (PNG) model. It belongs to the KPZ (KardarParisi-Zhang [19]) universality class and it can be described as follows (see Figure 1). At time $t$, the surface is described by an integer-valued height function $x \mapsto h(x, t) \in \mathbb{Z}, x \in \mathbb{R}, t \in \mathbb{R}_{+}$. It thus consists of up-steps $(\ulcorner)$ and down-steps $(\beth)$. The dynamics has a deterministic and a stochastic part:

(a) up- (down-) steps move to the left (right) with unit speed and disappear upon colliding,

*California Institute of Technology, e-mail: borodin@caltech.edu

${ }^{\dagger}$ Weierstrass Institute (WIAS), Berlin, e-mail: ferrari@wias-berlin.de

${ }_{\ddagger}^{\ddagger}$ Chiba University, e-mail: sasamoto@math.s.chiba-u.ac.jp 


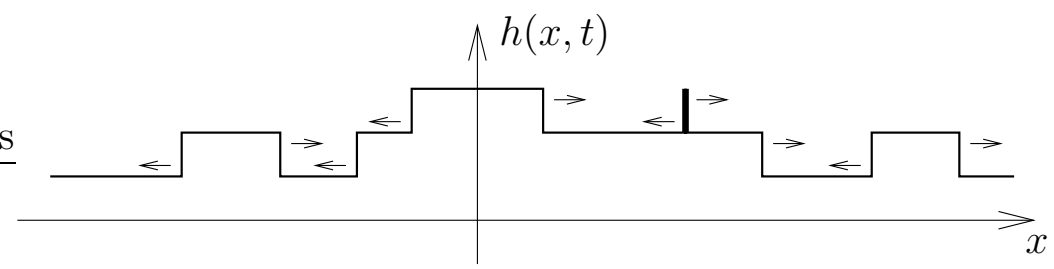

Figure 1: Illustration of the PNG height and its dynamics. The bold vertical piece is a nucleation. The arrows indicate the movements of the steps. A Java animation of the PNG dynamics is available at [9].

(b) pairs of up- and down- steps (nucleations) are randomly added on the surface with some given intensity.

The up- and down-steps of the nucleations then spread out with unit speed according to (a). The PNG model can be interpreted in several different ways, see [11] for a review.

On a macroscopic scale the surface of the PNG model grows deterministically, i.e., $\lim _{t \rightarrow \infty} t^{-1} h(\xi t, t)=H(\xi)$ is a non-random function. However, on a mesoscopic scale fluctuations grow in time. This is called roughening in statistical physics and extensive numerical studies have been made [3]. Since the PNG model is in the KPZ universality class, the fluctuation of the surface height is expected to live on a $t^{1 / 3}$ scale and non-trivial correlations are to be seen on a $t^{2 / 3}$ scale. Therefore, to have an interesting large time limit, we have to rescale the surface height as

$$
\frac{h\left(u t^{2 / 3}, t\right)-t H\left(u t^{-1 / 3}\right)}{t^{1 / 3}} .
$$

One of the most natural initial conditions for PNG is the flat initial condition, i.e., $h(x, 0)=0$ for all $x \in \mathbb{R}$. We consider nucleations occurring with translation-invariant intensity 1 We refer to the PNG model with such initial condition as flat PNG. In this case, by mapping the flat PNG to a point-to-line directed percolation model it was proven $[2,21,22]$ that the onepoint distribution is, in the $t \rightarrow \infty$ limit, the GOE Tracy-Widom distribution $F_{1}$, first discovered in random matrix theory [27]. However, no information on joint height distributions at more than one point has been previously known.

\footnotetext{
${ }^{1}$ In other words, the nucleation events form a Poisson process with constant intensity in the space-time upper half-plane.
} 
New Results. The main results of this paper are precisely the computation and asymptotic analysis of these joint distributions. In particular, we prove the convergence of the height rescaled as in (1.1) to the Airy 1 process in the $t \rightarrow \infty$ limit (see Section 2.2 for a definition of the process). The Airy 1 process has been discovered in the context of the asymmetric exclusion process $[5-7,24]$. Our result, stated in Theorem 6, is obtained by first determining an expression for the joint distributions for finite time $t$ (Proposition 4) and then taking the appropriate scaling limit.

Proposition 4 is actually just a particular case of Theorem 5, where we determine joint distributions along any space-like paths (as in Minkowski diagram), for which fixed time is a special case. The scaling limit is analyzed at this level of generality, thus Theorem 6 holds for any space-like paths. In contrast to previous works on the subject, our approach does not rely on the so-called RSK correspondence (RSK for Robinson-Schensted-Knuth), which was successfully applied for corner growth models, but does not seem to be well suited for the flat growth.

On the way of getting the results for the flat PNG, we consider a discrete time version of it, the Gates-Westcott dynamics [12,23]. This model is closely related to the totally asymmetric simple exclusion process (TASEP) in discrete time with parallel update and alternating initial conditions. The corresponding results for this model are Theorem 1 for the joint distributions along space-like paths, and Theorem 3 for the convergence to the Airy 1 process in the scaling limit. For the TASEP, the extreme situations of space-like paths are positions of different particles at a fixed time and positions of a fixed particle at different time moments (tagged particle). The space-like extension for TASEP is based on the previous paper [4].

Previous works on PNG. Another type of initial conditions for the PNG model has been analyzed before. It is the corner growth geometry, where nucleations occur only inside the cone $\{|x| \leq t\}$. The limit shape $H$ is a semi-circle, and the model is called $P N G$ droplet. In this geometry, the limit process has been obtained in [23]; it is known as Airy 2 process (previously called simply Airy process). The approach uses an extension to a multilayer model (inherited from the RSK construction), see [16,23]. The multilayer method was also used in other related models $[8,13,14,17,18,26]$. Also, for the flat PNG it was used to connect the associated point process at a single position and the point process of GOE eigenvalues [10]. Results on the behavior for the PNG droplet along space-like paths can be found in [8]. For a very brief description of the previously known results on TASEP fluctuations see the introductions of $[4,25]$. 


\section{Outline}

In section 2, we introduce our models and state the results. In section 3, we give an expression of the transition probability of the discrete TASEP as a marginal of a determinantal signed point process. In section 4 the Fredholm determinant expression for the joint distributions is obtained. The argument substantially relies on the algebraic techniques of [4]. In section 5, we consider the scaling limit of the parallel TASEP. In section 6, the continuous time PNG model is considered. In section 7, we consider the scaling limit for the continous PNG model.

\section{Acknowledgments}

A. Borodin was partially supported by the NSF grants DMS-0402047 and DMS-0707163. P.L. Ferrari is grateful to H. Spohn for useful discussions. The work of T. Sasamoto is supported by the Grant-in-Aid for Young Scientists (B), the Ministry of Education, Culture, Sports, Science and Technology, Japan.

\section{Models and results}

We start from the discrete time TASEP with parallel update. Then we will make the connection with a discrete version of the PNG, from which the continuous time PNG is obtained.

\subsection{Discrete time TASEP with parallel update}

We consider discrete time TASEP with parallel update and alternating initial conditions, i.e., particle $i$ has initial position $x_{i}(0)=-2 i, i \in \mathbb{Z}$. At each time step, each particle hops to its right neighbor site with probability $p=1-q$ provided that the site is empty. The particle positions at time $t$ is denoted by $x_{i}(t), i \in \mathbb{Z}$.

The dynamics of a particle depends only on particles on its right. This fact allows us to determine the joint distributions of particle positions also for different times, but restricted to "space-like paths". To define what we mean with "space-like paths", we consider a sequence of couples $\left(n_{i}, t_{i}\right)$, where $n_{i}$ is the number of the particle and $t_{i}$ is the time when this particle is observed. On such couples we define a partial order $\prec$, given by

$$
\left(n_{i}, t_{i}\right) \prec\left(n_{j}, t_{j}\right) \text { if } n_{j} \geq n_{i}, t_{j} \leq t_{i} \text {, and the two couples are not identical. }
$$


A space-like path is a sequence of ordered couples, namely,

$$
\mathcal{S}=\left\{\left(n_{k}, t_{k}\right), k=1,2, \ldots \mid\left(n_{k}, t_{k}\right) \prec\left(n_{k+1}, t_{k+1}\right)\right\} .
$$

The reason of the name "space-like" will be clear in the large time limit, where everything becomes continuous. Then space-like is the same concept as in the Minkowski diagram. The border cases for space-like paths are fixed time $\left(t_{i} \equiv t, \forall i\right)$ and fixed particle number $\left(n_{i} \equiv n, \forall i\right)$. The next theorem concerns the joint distributions of particle positions.

Theorem 1. Let particle with label $i$ start at $x_{i}(0)=-2 i, i \in \mathbb{Z}$. Consider a space-like path $\mathcal{S}$. For any given $m$, the joint distribution of the positions of the first $m$ points in $\mathcal{S}$ is given by

$$
\mathbb{P}\left(\bigcap_{k=1}^{m}\left\{x_{n_{k}}\left(t_{k}\right) \geq a_{k}\right\}\right)=\operatorname{det}\left(\mathbb{1}-\chi_{a}^{(-)} K \chi_{a}^{(-)}\right)_{\ell^{2}\left(\left\{\left(n_{1}, t_{1}\right), \ldots,\left(n_{m}, t_{m}\right)\right\} \times \mathbb{Z}\right)}
$$

where $\chi_{a}^{(-)}\left(\left(n_{k}, t_{k}\right), x\right)=\mathbb{1}\left(x<a_{k}\right)$. The kernel $K_{t}$ is given by

$$
\begin{aligned}
K\left(\left(n_{1}, t_{1}\right), x_{1} ;\left(n_{2}, t_{2}\right), x_{2}\right) & =-\phi^{\left(\left(n_{1}, t_{1}\right),\left(n_{2}, t_{2}\right)\right)}\left(x_{1}, x_{2}\right) \mathbb{1}_{\left[\left(n_{1}, t_{1}\right) \prec\left(n_{2}, t_{2}\right)\right]} \\
& +\widetilde{K}\left(\left(n_{1}, t_{1}\right), x_{1} ;\left(n_{2}, t_{2}\right), x_{2}\right),
\end{aligned}
$$

where

$$
\begin{aligned}
& \widetilde{K}\left(\left(n_{1}, t_{1}\right), x_{1} ;\left(n_{2}, t_{2}\right), x_{2}\right) \\
= & \frac{-1}{2 \pi \mathrm{i}} \oint_{\Gamma_{0}} \mathrm{~d} z \frac{(1+z)^{x_{2}+n_{1}+n_{2}}}{(-z)^{x_{1}+n_{1}+n_{2}+1}} \frac{(1-p)^{t_{1}-2 n_{1}-x_{1}}}{(1+p z)^{t_{1}+t_{2}+1-\left(x_{1}+n_{1}+n_{2}\right)}},
\end{aligned}
$$

and

$$
\begin{aligned}
& \phi^{\left(\left(n_{1}, t_{1}\right),\left(n_{2}, t_{2}\right)\right)}\left(x_{1}, x_{2}\right) \\
= & \frac{1}{2 \pi \mathrm{i}} \oint_{\Gamma_{-1}} \mathrm{~d} w \frac{(1+p w)^{t_{1}-t_{2}}}{(1+w)^{x_{1}-x_{2}+1}}\left(\frac{-w}{(1+w)(1+p w)}\right)^{n_{1}-n_{2}}
\end{aligned}
$$

where $\Gamma_{0}\left(r e s p . \Gamma_{-1}\right)$ is any simple loop, anticlockwise oriented, with 0 (resp. -1) being the unique pole of the integrand inside the contour.

Remark. In the limit $p \rightarrow 0$ under the time scaling by $p^{-1}$ the discrete time TASEP converges to the continuous time TASEP, and Theorem 1 turns into a special case of Proposition 3.6 of [4], where a more general continuous time model called PushASEP was considered. 


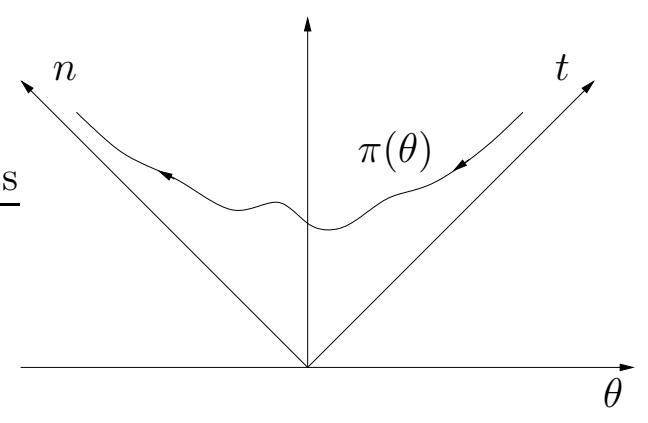

Figure 2: An example of a space-like path $\pi(\theta)$. Its slope is, in absolute value, at most 1 .

\section{2 $\quad$ Airy $_{1}$ process and scaling limit}

Starting from Theorem 1 we can analyze large time limits. The limit process is the so-called Airy, process introduced in $[6,24]$, which we recall here.

Definition 2 (The Airy 1 process). Define the extended kernel,

$$
\begin{aligned}
& K_{\mathcal{A}_{1}}\left(\tau_{1}, \xi_{1} ; \tau_{2}, \xi_{2}\right)=-\frac{1}{\sqrt{4 \pi\left(\tau_{2}-\tau_{1}\right)}} \exp \left(-\frac{\left(\xi_{2}-\xi_{1}\right)^{2}}{4\left(\tau_{2}-\tau_{1}\right)}\right) \mathbb{1}\left(\tau_{2}>\tau_{1}\right) \\
& +\operatorname{Ai}\left(\xi_{1}+\xi_{2}+\left(\tau_{2}-\tau_{1}\right)^{2}\right) \exp \left(\left(\tau_{2}-\tau_{1}\right)\left(\xi_{1}+\xi_{2}\right)+\frac{2}{3}\left(\tau_{2}-\tau_{1}\right)^{3}\right)
\end{aligned}
$$

The Airy process, $\mathcal{A}_{1}$, is the process with m-point joint distributions at $\tau_{1}<\tau_{2}<\ldots<\tau_{m}$ given by the Fredholm determinant,

$$
\mathbb{P}\left(\bigcap_{k=1}^{m}\left\{\mathcal{A}_{1}\left(\tau_{k}\right) \leq s_{k}\right\}\right)=\operatorname{det}\left(\mathbb{1}-\chi_{s} K_{\mathcal{A}_{1}} \chi_{s}\right)_{L^{2}\left(\left\{\tau_{1}, \ldots, \tau_{m}\right\} \times \mathbb{R}\right)}
$$

where $\chi_{s}\left(\tau_{k}, x\right)=\mathbb{1}\left(x>s_{k}\right)$.

Theorem 1 allows us to analyze joint distributions of particle positions for situations spanning between fixed time and fixed particle number (the tagged particle problem). One way to parametrize such situations is via a space-like path. We thus consider an arbitrary smooth function $\pi$ satisfying

$$
\left|\pi^{\prime}(\theta)\right| \leq 1 \text { and } \pi(\theta)+\theta>0
$$

see Figure 2. The requirement $\pi(\theta)+\theta>0$ reflects $t>0$. Then, we choose couples of $(t, n)$ on $\{((\pi(\theta)+\theta) T,(\pi(\theta)-\theta) T), \theta \in \mathbb{R}\}$, where $T$ is a large parameter. The case of fixed time, say $t=T$, is obtained by setting 
$\pi(\theta)=1-\theta$, while fixed particle number, say $n=\alpha T$, by $\pi(\theta)=\alpha+\theta$ with some constant $\alpha$.

From KPZ scaling exponents [19], we expect to see a nontrivial limit if we consider positions at distance of order $T^{2 / 3}$. Thus, the focus on the region around $\theta T$ is given by $\theta T-u T^{2 / 3}$, i.e., setting $\theta-u T^{-1 / 3}$ instead of $\theta$ and, by series expansions, we scale time and particle number as

$$
\begin{aligned}
t(u) & =\left\lfloor(\pi(\theta)+\theta) T-\left(\pi^{\prime}(\theta)+1\right) u T^{2 / 3}+\frac{1}{2} \pi^{\prime \prime}(\theta) u^{2} T^{1 / 3}\right\rfloor, \\
n(u) & =\left\lfloor(\pi(\theta)-\theta) T+\left(1-\pi^{\prime}(\theta)\right) u T^{2 / 3}+\frac{1}{2} \pi^{\prime \prime}(\theta) u^{2} T^{1 / 3}\right\rfloor .
\end{aligned}
$$

The KPZ fluctuation exponent is $1 / 3$, thus we expect to see fluctuations of particle positions on a scale of order $T^{1 / 3}$. Therefore, we define the rescaled process $\Xi_{T}$ by

$$
u \mapsto \Xi_{T}(u)=\frac{x_{n(u)}(t(u))-(-2 n(u)+\mathbf{v} t(u))}{-T^{1 / 3}} .
$$

Here the mean speed of particles, $\mathbf{v}$, is determined to be $\mathbf{v}=1-\sqrt{q}$ from the subsequent asymptotic analysis but can be known beforehand from the stationary measure for density $1 / 2$ [15]. This process has a limit as $T \rightarrow \infty$ given in terms of the Airy process. $^{2}$

Theorem 3. Let $\Xi_{T}$ be the rescaled process as in (2.11). Then

$$
\lim _{T \rightarrow \infty} \Xi_{T}(u)=\kappa_{\mathrm{v}} \mathcal{A}_{1}\left(\kappa_{\mathrm{h}} u\right),
$$

in the sense of finite dimensional distributions. The vertical (fluctuations) and horizontal (correlations) scaling coefficients are given by

$$
\begin{aligned}
& \kappa_{\mathrm{v}}=(\pi(\theta)+\theta)^{1 / 3}(1-q)^{1 / 3} q^{1 / 6}, \\
& \kappa_{\mathrm{h}}=\frac{(\pi(\theta)+\theta)^{2 / 3}(1-q)^{2 / 3} q^{-1 / 6}}{\left(\pi^{\prime}(\theta)+1\right)(1-\sqrt{q}) / 2+1-\pi^{\prime}(\theta)} .
\end{aligned}
$$

Remark. A similar result for the PushASEP with alternating initial condition has been proved in Theorem 2.2 of [4].

\subsection{TASEP and growth models}

As mentioned in the introduction, the discrete TASEP with parallel update is related to a surface growth model from which the polynuclear growth model in continuous time can be obtained as a limit. Let $t \geq 0$ and $x \in \mathbb{R}$ denote the time and the one-dimensional space coordinate respectively, and let $h_{t}(x)$ 


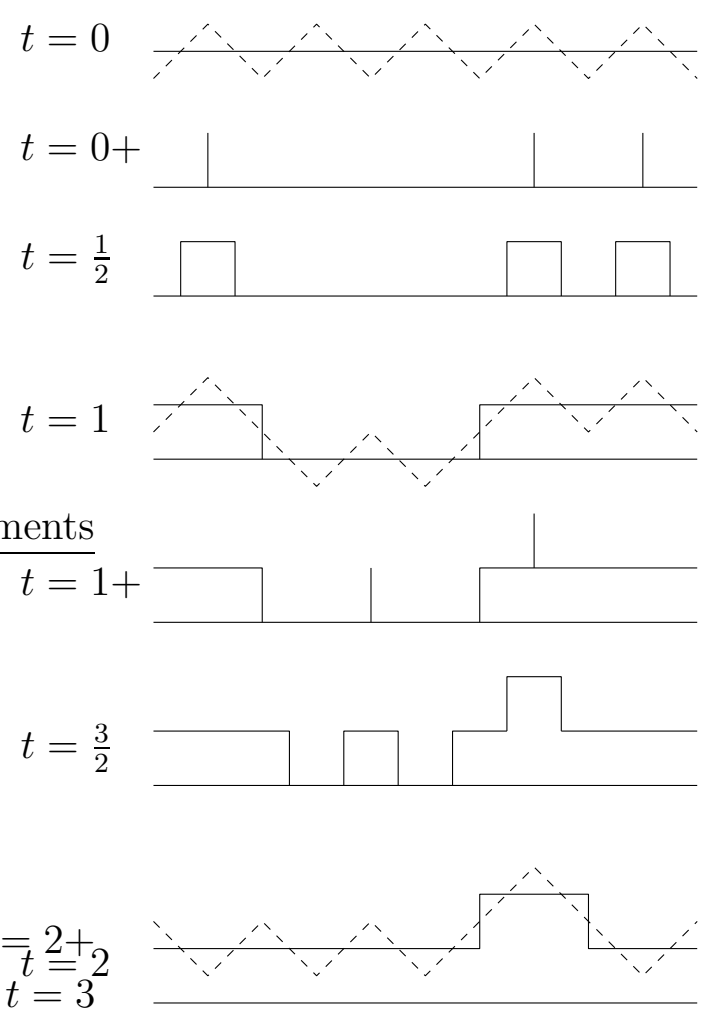

Figure 3: A surface growth model. For half-odd integer times this is equivalent to the discretized Gates-Westcott dynamics and for integer times to the discrete TASEP.

be the height of the surface at time $t$ and at position $x$. Let us introduce a dynamics of $h_{t}(x)$ as follows. Initially, at time $t=0$, the surface is flat; $h_{0}(x)=0$, for all $x \in \mathbb{R}$. Right after each integer time $(t=0+, 1+, 2+, \ldots)$, there could occur a nucleation with width 0 and height 1 with probability $q$ $(0<q<1)$ independently at each integer position $x$ such that $t+x+h_{t}(x)$ is even. Each nucleation is regarded as consisting of an upstep and a downstep and each upstep (resp. downstep) moves to the left (resp. right) with unit speed. This is a deterministic part of the evolution. When an upstep and a downstep collide, they merge together. See the solid line in Figure 3 for an example until $t=2$. The dynamics of the growth model, if we focus only on half-odd times $\left(t=\frac{1}{2}, \frac{3}{2}, \ldots\right)$, is the same as one considered in [23], i.e., a discretized version of the Gates-Westcott dynamics [12]. It is known that in an appropriate $q \rightarrow 0$ limit this growth model reduces to the standard continuous time PNG model [23].

To see the connection to the discrete TASEP, let us focus on integer 


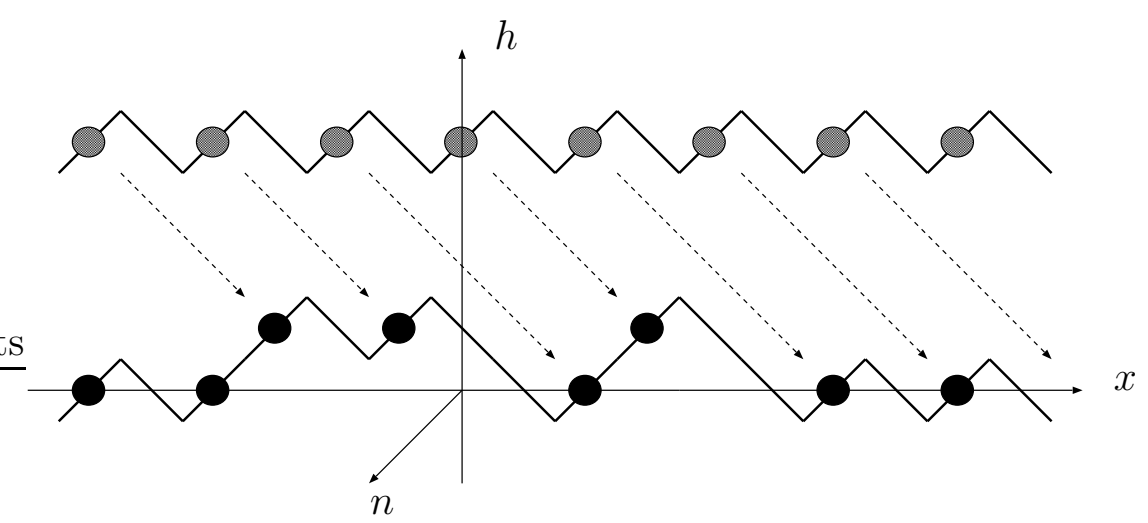

Figure 4: Surface height and TASEP particle positions. An expample for $t=4$.

times $(t=0,1,2, \ldots)$ and positions $(x \in \mathbb{Z})$ from now on and represent the surface as consisting of elementary upward slopes / and downward slopes $\backslash$ as indicated by dashed lines in Figure 3. At $t=0$, even (resp. odd) $x$ 's are taken to be the center of the upward (resp. downward) slopes. Then the dynamics of the surface is described as follows: At each time step the surface grows upward by unit height deterministically and then each local maximum $(\triangle)$ of slope turns into a local minimum $(\backslash /)$ independently with probability $p \equiv 1-q$. If we interpret an upward (resp. a downward) slope as a site occupied by a particle (resp. an empty site), this is equivalent to the discrete time TASEP with parallel update under the alternating initial condition.

The relation between the surface height $h_{t}(x)$ and the position of the TASEP particle is given by

$$
h_{t}(x) \leq H \Leftrightarrow x_{\left\lfloor\frac{t-x-H}{2}\right\rfloor}(t) \geq x
$$

and is understood as follows. On the plot of the surface at some fixed time $t$, draw also the initial surface at $h=t$. See Figure 4 for an expample. Then, from the correspondence between the growth model and the TASEP, the surface at time $t$ can be regarded as the particle positions. In this plot particles move along the down-right direction as indicated. The left hand side of $(2.15)$ is equivalent to the condition that the TASEP particle corresponding to $(x, h=t-H)$ has already reached $x$. Since the axis of the particle number $n$ is in the down-left direction, the value of $n$ corresponding to $(x, h=t-H)$ is $\lfloor(t-H-x) / 2\rfloor$. This consideration results in the relation (2.15). From the relation (2.15) the joint distributions of the height of the growth model 
is readily obtained through

$$
\mathbb{P}\left(\bigcap_{i=1}^{m}\left\{h_{t_{i}}\left(x_{i}\right) \leq H_{i}\right\}\right)=\mathbb{P}\left(\bigcap_{i=1}^{m}\left\{x_{n_{i}}\left(t_{i}\right) \geq x_{i}\right\}\right)
$$

combined with Theorem 1.

When $q \rightarrow 0$, the TASEP particles move almost deterministically and the surface $h_{t}(x)$ grows slowly, when a particle decides not to jump (with probability $q$ ). The continuous time PNG model is obtained by taking $q \rightarrow 0$ while setting space and time units to $\sqrt{q} / 2$ (the 2 is chosen to have nucleations with intensity 2 like in [23]). Denote by $\mathbf{x}$ and $\mathbf{t}$ the position and time variables in the continuous time PNG model. The PNG height function $h^{\mathrm{PNG}}(\mathbf{x}, \mathbf{t})$ is then obtained by the limit

$$
h^{\mathrm{PNG}}(\mathbf{x}, \mathbf{t})=\lim _{q \rightarrow 0} h_{2 \mathbf{t} / \sqrt{q}}(-2 \mathbf{x} / \sqrt{q}) .
$$

Here the minus sign on the right hand side is put for a convenience. The results below do not depend on this sign because of the symmetry of the model in consideration. The joint distribution of the surface height at time $\mathbf{t}$ is given as follows.

Proposition 4. Consider $m$ space positions $\mathbf{x}_{1}<\mathbf{x}_{2}<\ldots<\mathbf{x}_{m}$. Then, the joint distribution at time $\mathbf{t}$ of the heights $h^{\mathrm{PNG}}\left(\mathbf{x}_{k}, \mathbf{t}\right), k=1, \ldots, m$, is given by

$$
\mathbb{P}\left(\bigcap_{k=1}^{m}\left\{h^{\mathrm{PNG}}\left(\mathbf{x}_{k}, \mathbf{t}\right) \leq H_{k}\right\}\right)=\operatorname{det}\left(\mathbb{1}-\chi_{H} K_{\mathbf{t}}^{\mathrm{PNG}} \chi_{H}\right)_{\ell^{2}\left(\left\{\mathbf{x}_{1}, \ldots, \mathbf{x}_{m}\right\} \times \mathbb{Z}\right)}
$$

where the kernel is given by

$$
\begin{aligned}
& K_{\mathbf{t}}^{\mathrm{PNG}}\left(\mathbf{x}_{1}, h_{1} ; \mathbf{x}_{2}, h_{2}\right)=-I_{\left|h_{1}-h_{2}\right|}\left(2\left(\mathbf{x}_{2}-\mathbf{x}_{1}\right)\right) \mathbb{1}\left(\mathbf{x}_{2}>\mathbf{x}_{1}\right) \\
& +\left(\frac{2 \mathbf{t}+\mathbf{x}_{2}-\mathbf{x}_{1}}{2 \mathbf{t}-\mathbf{x}_{2}+\mathbf{x}_{1}}\right)^{\left(h_{1}+h_{2}\right) / 2} J_{h_{1}+h_{2}}\left(2 \sqrt{4 \mathbf{t}^{2}-\left(\mathbf{x}_{2}-\mathbf{x}_{1}\right)^{2}}\right) \mathbb{1}\left(2 \mathbf{t} \geq\left|\mathbf{x}_{2}-\mathbf{x}_{1}\right|\right)
\end{aligned}
$$

where $I_{n}(x)$ and $J_{n}(x)$ are the modified Bessel functions and the Bessel functions, see e.g. [1].

The last indicator function is obvious if one thinks about the PNG model. In fact, the height at position $\mathbf{x}$ at time $\mathbf{t}$ depends on events lying in the backward light cone of $(\mathbf{x}, \mathbf{t})$ on $\mathbb{R} \times \mathbb{R}_{+}$. Thus, when $\left|\mathbf{x}_{2}-\mathbf{x}_{1}\right|>2 \mathbf{t}$, the backwards light cones of $\left(\mathbf{x}_{1}, \mathbf{t}\right)$ and $\left(\mathbf{x}_{2}, \mathbf{t}\right)$ do not intersect in $\mathbb{R} \times \mathbb{R}_{+}$, which implies 
that the two height functions are independent. The Fredholm determinant then splits into blocks.

The result of Proposition 4 is actually a specialization of a more general situation which follows from the TASEP correspondence. In the TASEP, the space-like paths $\pi$ we had for particle numbers and times become the paths

$$
(\mathbf{x}, \mathbf{t})=(\pi(\theta)-3 \theta, \theta+\pi(\theta)) .
$$

The condition $\left|\pi^{\prime}(\theta)\right| \leq 1$ implies that $\partial \mathbf{t} / \partial \mathbf{x} \in[-1,0]$, i.e., these are spacelike paths as in special relativity oriented into the past. By the symmetry of the problem, one can consider also space-like paths locally oriented into the future, just looking at the process in the other direction.

Denote by $\gamma$ such a path on $\mathbb{R} \times \mathbb{R}_{+}^{*}$, i.e., $(\mathbf{x}, \mathbf{t}=\gamma(\mathbf{x}))$, then $\theta$ and $\pi(\theta)$ are given by the relations

$$
\theta=(\gamma(\mathbf{x})-\mathbf{x}) / 4, \quad \pi(\theta)=(3 \gamma(\mathbf{x})+\mathbf{x}) / 4,
$$

and the joint distributions of the surface height along the path $\gamma$ are expressed as in Theorem 5 ,

Theorem 5. Let us denote by $\mathbf{t}_{k}=\gamma\left(\mathbf{x}_{k}\right)$. Then, the joint distributions of $h^{\mathrm{PNG}}\left(\mathbf{x}_{k}, \mathbf{t}_{k}\right), k=1, \ldots, m$, is given by

$$
\mathbb{P}\left(\bigcap_{k=1}^{m}\left\{h^{\mathrm{PNG}}\left(\mathbf{x}_{k}, \mathbf{t}_{k}\right) \leq H_{k}\right\}\right)=\operatorname{det}\left(\mathbb{1}-\chi_{H} K^{\mathrm{PNG}} \chi_{H}\right)_{\ell^{2}\left(\left\{\left(\mathbf{x}_{1}, \mathbf{t}_{1}\right), \ldots,\left(\mathbf{x}_{k}, \mathbf{t}_{k}\right)\right\} \times \mathbb{Z}\right)}
$$

where the kernel is given by

$$
\begin{aligned}
& K^{\mathrm{PNG}}\left(\left(\mathbf{x}_{1}, \mathbf{t}_{1}\right), h_{1} ;\left(\mathbf{x}_{2}, \mathbf{t}_{2}\right), h_{2}\right)=-\left(\frac{\mathbf{x}_{2}-\mathbf{x}_{1}+\mathbf{t}_{1}-\mathbf{t}_{2}}{\mathbf{x}_{2}-\mathbf{x}_{1}-\mathbf{t}_{1}+\mathbf{t}_{2}}\right)^{\left(h_{1}-h_{2}\right) / 2} \\
& \times I_{\left|h_{1}-h_{2}\right|}\left(2 \sqrt{\left(\mathbf{x}_{2}-\mathbf{x}_{1}\right)^{2}-\left(\mathbf{t}_{2}-\mathbf{t}_{1}\right)^{2}}\right) \mathbb{1}_{\left\{\left(\mathbf{t}_{1}+\mathbf{x}_{1}, \mathbf{t}_{1}\right) \prec\left(\mathbf{t}_{2}+\mathbf{x}_{2}, \mathbf{t}_{2}\right)\right\}} \\
& +\left(\frac{\left(\mathbf{t}_{1}+\mathbf{t}_{2}\right)+\left(\mathbf{x}_{2}-\mathbf{x}_{1}\right)}{\left(\mathbf{t}_{1}+\mathbf{t}_{2}\right)-\left(\mathbf{x}_{2}-\mathbf{x}_{1}\right)}\right)^{\left(h_{2}+h_{1}\right) / 2} J_{h_{1}+h_{2}}\left(2 \sqrt{\left(\mathbf{t}_{1}+\mathbf{t}_{2}\right)^{2}-\left(\mathbf{x}_{2}-\mathbf{x}_{1}\right)^{2}}\right) \\
& \times \mathbb{1}\left(\mathbf{t}_{1}+\mathbf{t}_{2} \geq\left|\mathbf{x}_{1}-\mathbf{x}_{2}\right|\right)
\end{aligned}
$$

where $I_{n}(x)$ and $J_{n}(x)$ are the modified Bessel functions and the Bessel functions. The condition $\mathbb{1}_{\left\{\left(\mathbf{t}_{1}+\mathbf{x}_{1}, \mathbf{t}_{1}\right) \prec\left(\mathbf{t}_{2}+\mathbf{x}_{2}, \mathbf{t}_{2}\right)\right\}}$ means that $\mathbf{x}_{2}-\mathbf{x}_{1} \geq \mathbf{t}_{1}-\mathbf{t}_{2}>0$ or $\mathbf{x}_{2}-\mathbf{x}_{1}>\mathbf{t}_{1}-\mathbf{t}_{2} \geq 0$.

In the first term, for $\mathbf{x}_{2}>\mathbf{x}_{1}$, the condition $\mathbf{x}_{2}-\mathbf{x}_{1} \geq \mathbf{t}_{1}-\mathbf{t}_{2}$ is satisfied for $\mathbf{t}_{k}=\gamma\left(\mathbf{x}_{k}\right)$. Also, notice that when $\mathbf{x}_{2}-\mathbf{x}_{1} \rightarrow \mathbf{t}_{1}-\mathbf{t}_{2}$, the first term of the kernel goes to $\left(2\left(\mathbf{x}_{2}-\mathbf{x}_{1}\right)\right)^{\left|h_{1}-h_{2}\right|} /\left(\left|h_{1}-h_{2}\right|\right)$ ! . 


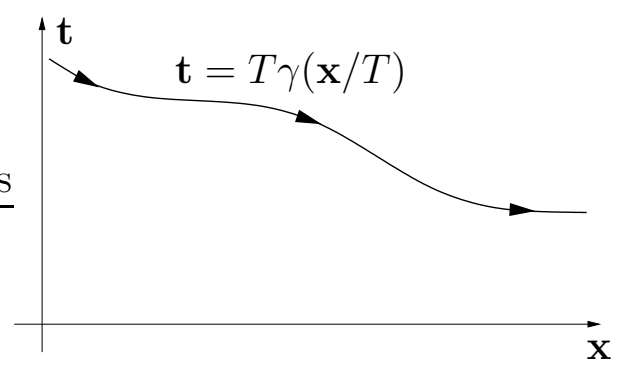

Figure 5: A space-time path $\gamma$ for continuous time PNG. $T$ is proportional to the PNG time $\mathbf{t}$.

\subsection{Scaling limit for the continuous PNG model}

The last result of this paper is the large time behavior of the flat PNG. The large parameter denoted by $T$ is proportional to time $\mathbf{t}$. Using the function $\gamma$, we consider $\mathbf{t}=T \gamma(\mathbf{x} / T)$, see Figure 5 .

Since the system is translation invariant, we focus around the origin, i.e., we look at the PNG height at

$$
\left\{\begin{array}{l}
\mathbf{x}(u)=u T^{2 / 3} \\
\mathbf{t}(u)=\gamma(0) T+\gamma^{\prime}(0) u T^{2 / 3}+\frac{1}{2} \gamma^{\prime \prime}(0) u^{2} T^{1 / 3} .
\end{array}\right.
$$

The surface height grows with the speed equal to 2. Thus, for large time $\mathbf{t}$, the macroscopic height will be close to $2 \mathbf{t}$. Fluctuations live on a $T^{1 / 3}$ scale. Consequently, we define the rescaled height process $h_{T}^{\mathrm{PNG}}$ by

$$
u \mapsto h_{T}^{\mathrm{PNG}}(u)=\frac{h^{\mathrm{PNG}}(\mathbf{x}(u), \mathbf{t}(u))-2 \mathbf{t}(u)}{T^{1 / 3}} .
$$

The large $T$ (thus large time too) behavior of $h_{T}^{\mathrm{PNG}}$ is given in terms of the Airy $_{1}$ process as stated below.

Theorem 6. Let $h_{T}^{\mathrm{PNG}}$ be the rescaled process as in (2.25). Then, in the limit of large $T$, we have

$$
\lim _{T \rightarrow \infty} h_{T}^{\mathrm{PNG}}(u)=S_{v} \mathcal{A}_{1}\left(S_{h} u\right),
$$

in the sense of finite dimensional distributions. The scaling coefficients $S_{v}$ and $S_{h}$ are given by

$$
S_{v}=(2 \gamma(0))^{1 / 3}, \quad S_{h}=(2 \gamma(0))^{2 / 3}=S_{v}^{2} .
$$

For $\gamma(x)=1$, i.e., fixed time, this was conjectured to hold in [6]. 


\section{Transition probability for the finite system}

Let $G\left(x_{1}, \ldots, x_{N} ; t\right)$ denote the transition probability of the parallel TASEP with $N$ particles starting at $t=0$ at positions $y_{N}<\ldots<y_{1}$. This is the probability that the $N$ particles starting from positions $y_{N}<\ldots<y_{1}$ at $t=0$ are at positions $x_{N}<\ldots<x_{1}$ at $t$.

Consider a determinantal signed point process on the set $\underline{x}=\left\{x_{i}^{n}, 1 \leq i \leq n \leq N\right\}$ by setting the measure

$W_{N}(\underline{x})=\left(\prod_{n=1}^{N-1} \operatorname{det}\left(\phi^{\sharp}\left(x_{i}^{n}, x_{j+1}^{n+1}\right)\right)_{0 \leq i, j \leq n}\right) \operatorname{det}\left(F_{-i+1}\left(x_{j}^{N}-y_{N+1-i}, t+1-i\right)\right)_{1 \leq i, j \leq N}$

where

$$
\phi^{\sharp}(x, y)= \begin{cases}1, & y \geq x, \\ p, & y=x-1, \\ 0, & y \leq x-2,\end{cases}
$$

the function $F_{n}(x, t)$ defined by

$$
F_{-n}(x, t)=\frac{1}{2 \pi \mathrm{i}} \oint_{\Gamma_{0,-1}} \mathrm{~d} w \frac{w^{n}}{(1+w)^{n+x+1}}(1+(1-q) w)^{t},
$$

and where we used the convention, $x_{0}^{n}=-\infty$.

The following proposition states that the one time transition probability of the TASEP is given as a marginal of the signed measure (3.1).

Proposition 7. Let us set $x_{1}^{n}=x_{n}, n=1, \ldots, N$. Then

$$
G\left(x_{1}, \ldots, x_{N} ; t\right)=\sum_{\mathcal{D}} W_{N}(\underline{x})
$$

where summation is over the variables in the set,

$$
\mathcal{D}=\left\{x_{i}^{n}, 2 \leq i \leq n \leq N \mid x_{i}^{n}>x_{i-1}^{n}\right\}
$$

varying over $\mathbb{Z}$.

Note that $W_{N}(\underline{x})$ is actually symmetric with respect to permutations of variables with same upper index, so the ordering in (3.5) is used for singling out the minimal $x_{1}^{n}=\min \left\{x_{i}^{n}, i=1, \ldots, n\right\}$.

Remark. Similar representations for the transition probability of continuous time TASEP, discrete time TASEP with sequential update and PushASEP have been obtained in [4-6].

In the different parts of the proof of Proposition 1, we will use several properties of the function $F_{n}$, which are listed below. 


\section{Lemma 8.}

$$
\begin{aligned}
F_{n+1}(x, t) & =\sum_{y=x}^{\infty} F_{n}(y, t), \\
F_{n}(x, t+1) & =q F_{n}(x, t)+(1-q) F_{n}(x-1, t) \\
& =F_{n}(x, t)+(1-q) F_{n-1}(x-1, t), \\
\left(\phi^{\sharp} * F_{n}\right)(x, t) & =F_{n+1}(x, t+1), \\
F_{-n}(x,-n) & =0 \quad \text { for } x<-n, n>0, \\
F_{n}(x, n) & =0 \text { for } x>n, n>0, \\
F_{n}(n, n) & =(1-q)^{n}, n \geq 0, \\
F_{-n}(-n,-n) & =1 /(-q)^{n}, n \geq 0 .
\end{aligned}
$$

Here “*” represents the convolution: $\left(\phi^{\sharp} * f\right)(x)=\sum_{y} \phi^{\sharp}(x, y) f(y)$.

Proof of Lemma 8. These are proven by using the definition (3.2) and (3.3).

The first step in the proof of Proposition 7 is the following Lemma.

Lemma 9. Let us set

$$
\phi_{\nu}^{\sharp}(x, y)= \begin{cases}\nu^{y-x}, & y \geq x, \\ 1-q, & y=x-1, \\ 0, & y \leq x-2\end{cases}
$$

and $\phi_{\nu}^{\sharp}(-\infty, y)=\nu^{y}$. Then, for any antisymmetric function $f\left(b_{1}, \ldots, b_{n}\right)$,

$$
\begin{aligned}
& \sum_{\substack{b_{n}>\ldots>b_{1}>b_{0} \\
b_{0}: \text { fixed }}} \operatorname{det}\left(\phi_{\nu}^{\sharp}\left(a_{i}, b_{j}\right)\right)_{0 \leq i, j \leq n} \cdot f\left(b_{1}, \ldots, b_{n}\right) \\
= & g_{\nu}\left(a_{1}, b_{0}\right) \sum_{\substack{b_{n}>\ldots>b_{1}>b_{0} \\
b_{0}: \text { fixed }}} \operatorname{det}\left(\phi_{\nu}^{\sharp}\left(a_{i}, b_{j}\right)\right)_{1 \leq i, j \leq n} \cdot f\left(b_{1}, \ldots, b_{n}\right)
\end{aligned}
$$

where $a_{n}>\ldots>a_{1}, a_{0}=-\infty$ and

$$
g_{\nu}(a, b)= \begin{cases}0, & b \geq a \\ \nu^{b}(1-(1-q) \nu), & b=a-1 \\ \nu^{b}, & b \leq a-2\end{cases}
$$


Proof of Lemma 9. From the antisymmetry of $f$ and of the determinant, (3.15) is equivalent to

$$
\begin{aligned}
& \sum_{\substack{b_{1}, \ldots, b_{n}>b_{0} \\
b_{0}: \text { fixed }}} \operatorname{det}\left(\phi_{\nu}^{\sharp}\left(a_{i}, b_{j}\right)\right)_{0 \leq i, j \leq n} \cdot f\left(b_{1}, \ldots, b_{n}\right) \\
= & g_{\nu}\left(a_{1}, b_{0}\right) \sum_{\substack{b_{1}, \ldots, b_{n}>b_{0} \\
b_{0} \text { :fixed }}} \operatorname{det}\left(\phi_{\nu}^{\sharp}\left(a_{i}, b_{j}\right)\right)_{1 \leq i, j \leq n} \cdot f\left(b_{1}, \ldots, b_{n}\right) .
\end{aligned}
$$

Since a basis of the antisymmetric functions is made of the antisymmetric delta functions and the relation to prove is linear in $f$, it is enough to consider

$$
f\left(b_{1}, \ldots, b_{n}\right)= \begin{cases}(-1)^{\sigma}, & \text { if }\left(b_{1}, \ldots, b_{n}\right)=\left(b_{\sigma_{1}}, \ldots, b_{\sigma_{n}}\right) \text { for some } \sigma \in S_{n} \\ 0, & \text { otherwise }\end{cases}
$$

for fixed $b_{1}, \ldots, b_{n}>b_{0}$. Here $S_{n}$ is the group of all permutations of $\{1, \ldots, n\}$. For this special choice of $f$, the left hand side of (3.17) is $n$ ! times the single determinant,

$$
\operatorname{det}\left[\begin{array}{cccc}
\nu^{b_{0}} & \nu^{b_{1}} & \ldots & \nu^{b_{n}} \\
\phi_{\nu}^{\sharp}\left(a_{1}, b_{0}\right) & \phi_{\nu}^{\sharp}\left(a_{1}, b_{1}\right) & \ldots & \phi_{\nu}^{\sharp}\left(a_{1}, b_{n}\right) \\
\vdots & \vdots & & \vdots \\
\phi_{\nu}^{\sharp}\left(a_{n}, b_{0}\right) & \phi_{\nu}^{\sharp}\left(a_{n}, b_{1}\right) & \ldots & \phi_{\nu}^{\sharp}\left(a_{n}, b_{n}\right)
\end{array}\right] .
$$

We have the following three cases.

(a) $a_{1} \leq b_{0}$ : the second row gives $\left(\nu^{b_{0}-a_{1}}, \ldots, \nu^{b_{n}-a_{1}}\right)$ which is proportional to the first row. Therefore in this case the LHS is zero.

(b) $a_{1}=b_{0}+1$ : The second row is $\left(1-q, \nu^{b_{1}-a_{1}}, \ldots, \nu^{b_{n}-a_{1}}\right)$. Subtracting $\nu^{a_{1}}$ times the second row from the first row one obtains

$$
\nu^{b_{0}}(1-(1-q) \nu) \cdot \operatorname{det}\left(\phi_{\nu}^{\sharp}\left(a_{i}, b_{j}\right)\right)_{1 \leq i, j \leq n} .
$$

(c) $a_{1}>b_{0}+1$ : The first column is $\left(\nu^{b_{0}}, 0, \ldots, 0\right)^{t}$. Thus the determinant is $\nu^{b_{0}} \cdot \operatorname{det}\left(\phi_{\nu}^{\sharp}\left(a_{i}, b_{j}\right)\right)_{1 \leq i, j \leq n}$.

The result in each case agrees with $n$ ! times the RHS of (3.17) and hence the lemma is proved.

Let $\mathcal{N}\left(x_{1}, \ldots, x_{N}\right)$ denote the number of $j$ 's s.t. $x_{j}-x_{j+1}=1, j=$ $1, \ldots, N-1$. Using the above lemma with $\nu=1$ in which case $\phi_{\nu}^{\sharp}$ reduces to $\phi^{\sharp}$, we have the following result. 
Lemma 10. With $x_{1}^{n}=x_{n}, n=1, \ldots, N$, one has

$$
\begin{aligned}
\sum_{\mathcal{D}} W_{N}(\underline{x}) & =q^{\mathcal{N}\left(x_{1}, \ldots, x_{N}\right)} \operatorname{det}\left[F_{j-i}\left(x_{N-j+1}-y_{N-i+1}, t+j-i\right)\right]_{1 \leq i, j \leq N} \\
& =: \widetilde{G}\left(x_{1}, \ldots, x_{N} ; t\right) .
\end{aligned}
$$

Proof of Lemma 10. For simplicity, we denote

$$
f_{i}(x)=F_{-i+1}\left(x-y_{N-i+1}, t-i+1\right),
$$

for $i=1, \ldots, N$. From the definitions (3.1), the LHS of (3.21) writes

$$
\sum_{\substack{x_{n}^{n}>x_{n-1}^{n}>\ldots>x_{1}^{n} \\ x_{1}^{n} \text { :fixed,1 } 1 \leq n \leq N}}\left(\prod_{n=1}^{N-1} \operatorname{det}\left(\phi^{\sharp}\left(x_{i}^{n}, x_{j+1}^{n+1}\right)\right)_{0 \leq i, j \leq n}\right) \operatorname{det}\left(f_{i}\left(x_{j}^{N}\right)\right)_{1 \leq i, j \leq N} .
$$

Applying Lemma 9 with $\nu=1, n=N-1, a_{i}=x_{i}^{N-1}, i=1, \ldots, N-1$, $b_{i}=x_{i+1}^{N}, i=0, \ldots, N-1$ and

$$
f\left(b_{1}, \ldots, b_{n}\right)=\operatorname{det}\left(f_{i}\left(x_{j}^{N}\right)\right)_{1 \leq i, j \leq N}
$$

we obtain

$$
\begin{aligned}
(\underline{3.23})= & g_{1}\left(x_{1}^{N-1}, x_{1}^{N}\right) \cdot \sum_{\substack{x_{n}^{n}>x_{n-1}^{n}>\ldots>x_{1}^{n} \\
x_{1}^{n}: \text { fixed }, 1 \leq n \leq N-1}}\left(\prod_{n=1}^{N-2} \operatorname{det}\left(\phi^{\sharp}\left(x_{i}^{n}, x_{j+1}^{n+1}\right)\right)_{0 \leq i, j \leq n}\right) \\
& \times \sum_{\substack{x_{N}^{N}>x_{N-1}^{N}>\ldots>x_{1}^{N} \\
x_{1}^{N}: \text { fixed }}} \operatorname{det}\left(\phi^{\sharp}\left(x_{i}^{N-1}, x_{j+1}^{N}\right)\right)_{1 \leq i, j \leq N-1} \cdot \operatorname{det}\left(f_{i}\left(x_{j}^{N}\right)\right)_{1 \leq i, j \leq N} .
\end{aligned}
$$

Heine's identity,

$$
\frac{1}{n !} \sum_{x_{1}, \ldots, x_{n}} \operatorname{det}\left(\varphi_{i}\left(x_{j}\right)\right)_{1 \leq i, j \leq n} \operatorname{det}\left(\psi_{i}\left(x_{j}\right)\right)_{1 \leq i, j \leq n}=\operatorname{det}\left[\phi_{i} * \psi_{j}\right]_{1 \leq i, j \leq n}
$$

allows us to rewrite the last summation in (3.25) as

$$
\operatorname{det}\left[\begin{array}{cccc}
f_{1}\left(x_{1}^{N}\right) & \left(\phi^{\sharp} * f_{1}\right)\left(x_{1}^{N-1}\right) & \ldots & \left(\phi^{\sharp} * f_{1}\right)\left(x_{N-1}^{N-1}\right) \\
\vdots & \vdots & & \vdots \\
f_{N}\left(x_{1}^{N}\right) & \left(\phi^{\sharp} * f_{N}\right)\left(x_{1}^{N-1}\right) & \ldots & \left(\phi^{\sharp} * f_{N}\right)\left(x_{N-1}^{N-1}\right)
\end{array}\right] .
$$


We repeat the procedure up to a total of $j-1$ times in column $j$ and we get

$$
(\underline{3.25})=\left(\prod_{n=1}^{N-1} g_{1}\left(x_{1}^{n}, x_{1}^{n+1}\right)\right) \operatorname{det}[\overbrace{\phi^{\sharp} * \ldots * \phi^{\sharp}}^{j-1} * f_{i}\left(x_{1}^{N-j+1}\right)]_{1 \leq i, j \leq N} .
$$

The proof of the lemma is finished using (3.22), (3.9) and $\prod_{n=1}^{N-1} g_{1}\left(x_{1}^{n}, x_{1}^{n+1}\right)=q^{\mathcal{N}\left(x_{1}, \ldots, x_{N}\right)}$.

Proof of Proposition 7 . We need to prove

$$
G\left(x_{1}, \ldots, x_{N} ; t\right)=\widetilde{G}\left(x_{1}, \ldots, x_{N} ; t\right) .
$$

This statement was also proved in [20] by the Bethe ansatz techniques. Our proof is by induction in $t$. We start by showing that the initial conditions agree, i.e., $\widetilde{G}\left(x_{1}, \ldots, x_{N} ; 0\right)=G\left(x_{1}, \ldots, x_{N} ; 0\right)$, that is,

$$
q^{\mathcal{N}\left(x_{1}^{1}, \ldots, x_{1}^{N}\right)} \cdot \operatorname{det}\left[F_{j-i}\left(x_{N-j+1}-y_{N-i+1}, j-i\right)\right]_{1 \leq i, j \leq N}=\prod_{n=1}^{N} \delta_{x_{n}, y_{n}} .
$$

We first show that LHS of (3.30) is zero if $x_{N} \neq y_{N}$. If $x_{N} \leq y_{N}-1$, since $y_{N-i+1} \geq y_{N}+i-1$, one has $x_{N}-y_{N-i+1}<-i+1, i=1, \ldots, N$. Then, from (3.10) we have $F_{1-i}\left(x_{N}-y_{N-i+1}, 1-i\right)=0$, i.e., the first column of LHS of (3.30) is zero. Similarly, if $x_{N} \geq y_{N}+1$, since $x_{N-j+1} \geq x_{N}+j-1$, one has $x_{N-j+1}-y_{N}>j-1, j=1, \ldots, N$. Then, from (3.11) we have $F_{j-1}\left(x_{N-j+1}-y_{N}, j-1\right)=0$, i.e., the first row of LHS of (3.30) is zero. This agrees with RHS of (3.30) also being zero if $x_{N} \neq y_{N}$.

Now let us assume $x_{N}=y_{N}$. There are two cases.

(a) $y_{N-1}>y_{N}+1$. In this case, since $x_{N}-y_{N-i+1}=y_{N}-y_{N-i+1}<-i+1, i=$ $2, \ldots, N$, one has $F_{1-i}\left(x_{N}-y_{N-i+1}, 1-i\right)=0, i=2, \ldots, N$. Then the first column of LHS of (3.30) is $(1,0, \ldots, 0)^{t}$ and hence the determinant is equal to $\operatorname{det}\left[F_{j-i}\left(x_{N-j+1}-y_{N-i+1}, j-i\right)\right]_{2 \leq i, j \leq N}$.

(b) $y_{N-1}=y_{N}+1$. First let us see that LHS of (3.30) is zero when $x_{N-1} \neq$ $y_{N-1}$. We have $x_{N-1} \geq x_{N}+1=y_{N}+1=y_{N-1}$. If $x_{N-1} \geq y_{N-1}+1$, we have $x_{N-j+1}-y_{N} \geq x_{N-1}+j-2-\left(y_{N-1}-1\right) \geq j$, for $j=2, \ldots, N$, and $x_{N-j+1}-y_{N-1} \geq j-1$, for $j=2, \ldots, N$. Then the first and the second row of LHS of (3.30) are both of the form, $(*, 0, \ldots, 0)$ where $*$ represents an arbitrary number and hence the determinant is zero. Hence LHS of (3.30) is zero if $x_{N-1} \neq y_{N-1}$. On the other hand, when $x_{N-1}=y_{N-1}$, the upper-left $2 \times 2$ submatrix of the determinant is

$$
\left[\begin{array}{cc}
F_{0}(0,0) & F_{1}(1,1) \\
F_{-1}(-1,-1) & F_{0}(0,0)
\end{array}\right]=\left[\begin{array}{cc}
1 & 1-q \\
-1 / q & 1
\end{array}\right],
$$


whose determinant is $1 / q$.

Repeating the same procedure, at each step one has either case (a) or (b). The final result is that $y_{k}=x_{k}$, for $k=1, \ldots, N$, otherwise the determinant in LHS of (3.30) is zero. Moreover, when $y_{k}=x_{k}, k=1, \ldots, N$, denote by $n_{1}, n_{1}+n_{2}, \ldots, n_{1}+\ldots+n_{\ell}$ the values of $j$ such that $x_{j-1}-x_{j}>1$. Then LHS of (3.30) is equal to $\prod_{m=1}^{\ell} D_{n_{m}}$ with

$$
D_{n}=\operatorname{det}\left[F_{j-i}(j-i, j-i)\right]_{1 \leq i, j \leq n}
$$

Finally using (3.12), (3.13), we obtain an explicit form of the matrix. To compute its determinant it is enough to develop along the first row. The determinant of the $(1,1)$ minor is $D_{n-1}$, while the one of the $(1,2)$ minor is $(-1 / q) D_{n-1}$ because the minor is the same as the $(1,1)$ minor except the first column is multiplied by $-1 / q$. All the other minors have determinant zero, because the first two column are linearly dependent. Thus, $D_{n}=$ $1 \cdot D_{n-1}-(1-q) /(-q) D_{n-1}$, and since $D_{1}=1$, it follows that

$$
D_{n}=\frac{1}{q^{n-1}}
$$

This ends the part of the proof concerning initial conditions.

Next we prove that (3.29) holds for $t+1$ if it does for $t$. Since this is true for $t=0$, by induction it will be true for all $t \in \mathbb{N}$. $G$ satisfies the TASEP dynamics, thus

$$
\begin{aligned}
& G\left(x_{1}, \ldots, x_{N} ; t+1\right) \\
= & \sum_{z} G\left(z_{1}, \ldots, z_{N}, t\right) w(z, x)=\sum_{z} \widetilde{G}\left(z_{1}, \ldots, z_{N}, t\right) w(z, x) \\
= & \sum_{z} w(z, x) q^{\mathcal{N}\left(z_{1}, \ldots, z_{N}\right)} \operatorname{det}\left[F_{j-i}\left(z_{N-j+1}-y_{N-i+1}, t+j-i\right)\right]_{1 \leq i, j \leq N} .
\end{aligned}
$$

Here

$$
w(z, x)=\prod_{n=1}^{N} v_{n}, \quad v_{n}= \begin{cases}1, & z_{n}=z_{n-1}-1, x_{n}=z_{n}, \\ q, & z_{n}<z_{n-1}-1, x_{n}=z_{n} \\ 1-q, & z_{n}<z_{n-1}-1, x_{n}=z_{n}+1\end{cases}
$$

and in the second equality we have used the assumption of the induction. We rewrite $\widetilde{G}\left(x_{1}, \ldots, x_{N} ; t+1\right)$ using (3.7) and (3.8) as follows. For $k$ from 1 to $N$ :

(a) if $x_{k}=x_{k+1}+1$, then we use (3.8) to the $N+1-k$ th column. Then, the new term with the $(1-q)$ factor in front cancels out because it is proportional 


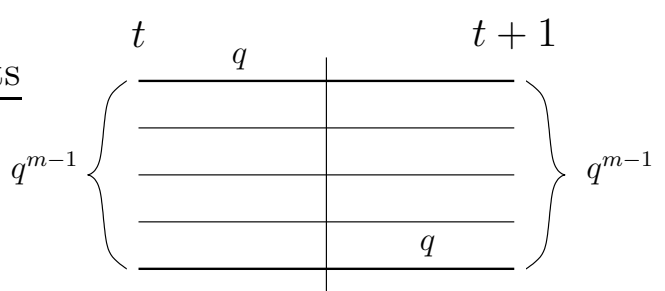

(a)

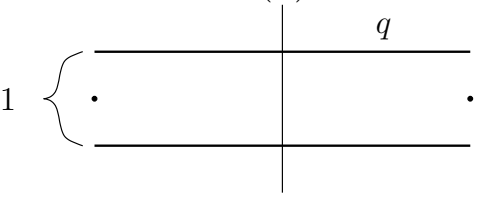

$(c)$

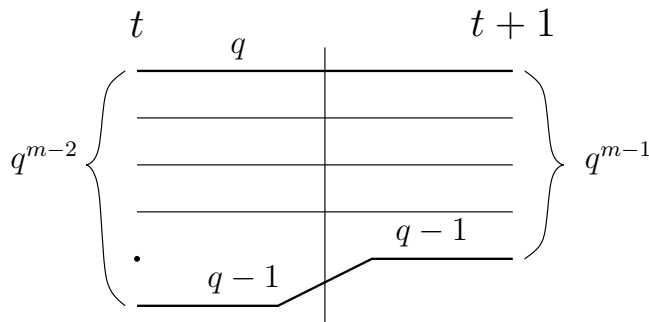

(b)

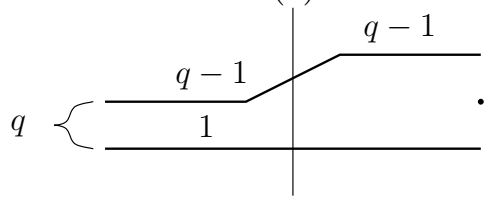

$(d)$

Figure 6: Graphical representation of (3.38). The dots represents empty places, while a line leaving/arriving to a point is an occupied position. In (a) and (b), on the left (resp. right) we indicate the weights different from 1 of LHS (resp. RHS) of (3.38). In (c) and (d) the bottom and top lines of two blocks at distance 2 at time $t+1$ are represented, for the cases corresponding to (a) and (b) for the top block.

to its left column of the determinant.

(b) if $x_{k}>x_{k+1}+1$, then we just use (3.7).

With these replacements we get

$$
\begin{aligned}
& \widetilde{G}\left(x_{1}, \ldots, x_{N} ; t+1\right) \\
= & \sum_{z} \tilde{w}(z, x) q^{\mathcal{N}\left(x_{1}, \ldots, x_{N}\right)} \operatorname{det}\left[F_{j-i}\left(z_{N-j+1}-y_{N-i+1}, t+j-i\right)\right]_{1 \leq i, j \leq N},
\end{aligned}
$$

where

$$
\tilde{w}(z, x)=\prod_{n=1}^{N} \tilde{v}_{n}, \quad \tilde{v}_{n}= \begin{cases}1, & x_{n}=x_{n+1}+1, z_{n}=x_{n}, \\ q, & x_{n}>x_{n+1}+1, z_{n}=x_{n}, \\ 1-q, & x_{n}>x_{n+1}+1, z_{n}=x_{n}-1 .\end{cases}
$$

Comparing (3.34) and (3.36), it is enough to show

$$
q^{\mathcal{N}\left(z_{1}, \ldots, z_{N}\right)} w(z, x)=q^{\mathcal{N}\left(x_{1}, \ldots, x_{N}\right)} \tilde{w}(z, x) .
$$

This indeed holds and can be seen by checking case by case. We illustrate it using Figure 6. First consider a block of particles, say $m$ of them at time 
$t+1$. There are two possibility of reaching this situations in one time step, as indicated in Figure 6 (a) and (b). The products of all the weights on the right and on the left are the same, i.e., (3.38) holds for a single block of particles. If two blocks of particles at time $t+1$ are at distance at least 2 , they are independent during one time step. We just have to check that (3.38) holds for two blocks at distance 2 at time $t+1$. Case (a) is illustrated in (c) and the weights are unchanged for both blocks. Case (b) is illustrated in (d). This time, the $q$ on the top line of the second block becomes a 1 , but this is compensated by an extra factor $q$ on the left.

\section{Joint distributions along space-like paths}

Theorem 11. Let us consider particles starting from $y_{1}>y_{2}>\ldots$ and denote $x_{j}(t)$ the position of $j$ th particle at time $t$. Consider a sequences of particles and times which are space-like, i.e., a sequence of $m$ such couples $\mathcal{S}=\left\{\left(n_{k}, t_{k}\right), k=1, \ldots, m \mid\left(n_{k}, t_{k}\right) \prec\left(n_{k+1}, t_{k+1}\right)\right\}$. The joint distribution of their positions $x_{n_{k}}\left(t_{k}\right)$ is given by

$$
\mathbb{P}\left(\bigcap_{k=1}^{m}\left\{x_{n_{k}}\left(t_{k}\right) \geq a_{k}\right\}\right)=\operatorname{det}\left(\mathbb{1}-\chi_{a} K \chi_{a}\right)_{\ell^{2}\left(\left\{\left(n_{1}, t_{1}\right), \ldots,\left(n_{m}, t_{m}\right)\right\} \times \mathbb{Z}\right)}
$$

where $\chi_{a}\left(\left(n_{k}, t_{k}\right), x\right)=\mathbb{1}\left(x<a_{k}\right)$. Here $K$ is the extended kernel with entries

$K\left(\left(n_{1}, t_{1}\right), x_{1} ;\left(n_{2}, t_{2}\right), x_{2}\right)=-\phi^{*\left(\left(n_{1}, t_{1}\right),\left(n_{2}, t_{2}\right)\right)}\left(x_{1}, x_{2}\right)+\sum_{k=1}^{n_{2}} \Psi_{n_{1}-k}^{n_{1}, t_{1}}\left(x_{1}\right) \Phi_{n_{2}-k}^{n_{2}, t_{2}}\left(x_{2}\right)$

where

$$
\begin{aligned}
& \phi^{*\left(\left(n_{1}, t_{1}\right),\left(n_{2}, t_{2}\right)\right)}\left(x_{1}, x_{2}\right) \\
= & \frac{1}{2 \pi \mathrm{i}} \oint_{\Gamma_{0,-1}} \mathrm{~d} w \frac{(1+p w)^{t_{1}-t_{2}}}{(1+w)^{x_{1}-x_{2}+1}}\left(\frac{w}{(1+w)(1+p w)}\right)^{n_{1}-n_{2}} \mathbb{1}_{\left[\left(n_{1}, t_{1}\right) \prec\left(n_{2}, t_{2}\right)\right]},
\end{aligned}
$$

and the functions $\Psi_{n-l}^{n, t}$ are given by

$$
\Psi_{n-k}^{n, t}(x)=\frac{1}{2 \pi \mathrm{i}} \oint_{\Gamma_{0,-1}} \mathrm{~d} w \frac{(1+p w)^{t}}{(1+w)^{x-y_{k}+1}}\left(\frac{w}{(w+1)(1+p w)}\right)^{n-k} .
$$

The functions $\Phi_{n-k}^{n, t}$ are determined by the two conditions

$$
\sum_{x \in \mathbb{Z}} \Psi_{n-l}^{n, t}(x) \Phi_{n-k}^{n, t}(x)=\delta_{k, l}, \quad 1 \leq k, l \leq n
$$


and

$$
\operatorname{span}\left\{\Phi_{n-k}^{n, t}(x), k=1, \ldots, n\right\}=\operatorname{span}\left\{1, x, \ldots, x^{n-1}\right\} .
$$

The paths $\Gamma_{0,-1}$ are any simple loops anticlockwise oriented including $0,-1$ and no other poles.

Proof of Theorem 11. This is the analogue of Proposition 3.1 of [4]. The first step to obtain this proposition, was Lemma 4.4 of [4]. In the simple case where in the model studied in [4] all particles have the same jump rates, i.e., $v_{i}=1$ for all $i$, then Lemma 4.4 of [4] and our Proposition 7 have exactly the same structure. For the comparison of the two, we need just the following identifications.

\begin{tabular}{|c|c|}
\hline PushASEP & Parallel TASEP \\
\hline$\varphi(x, y)$ & $\phi^{\sharp}(x, y)$ \\
$F_{n}(x, a, b)$ & $\widetilde{F}_{n}(x, t)$ \\
\hline
\end{tabular}

where

$$
\widetilde{F}_{n}(x, t)=F_{n}(x, t+n) .
$$

From (3.9) it then follows that $\widetilde{F}_{n}$ satisfies

$$
\left(\phi^{\sharp} * \widetilde{F}_{n}\right)(x, t)=\widetilde{F}_{n+1}(x, t) .
$$

The identity (4.8) corresponds to Lemma 4.3 of [4]. To obtain Proposition 3.1 of [4] one needs to prove Lemma 4.5 of [4] which needs as ingredients only Lemma 4.3 of [4]. These Lemmas are the only ingredients used to obtain Theorem 4.1 and then Theorem 4.2 of [4]. This last theorem concerns some general determinantal measures (a generalization of Lemma 3.4 in [6]), whose specialization to the PushASEP model is Proposition 3.1 of [4]. All these steps go through unchanged in our case provided we make the above identifications.

To obtain the precise expressions of Theorem 11, we use an integral representation for $\phi^{\sharp}$, namely,

$$
\phi^{\sharp}(x, y)=\frac{1}{2 \pi \mathrm{i}} \oint_{\Gamma_{0,-1}} \mathrm{~d} w \frac{1}{(w+1)^{x-y+1}} \frac{(1+p w)(1+w)}{w} .
$$

From this we get

$$
\left(\phi^{\sharp}\right)^{n}(x, y)=\frac{1}{2 \pi \mathrm{i}} \oint_{\Gamma_{0,-1}} \mathrm{~d} w \frac{1}{(w+1)^{x-y+1}}\left(\frac{(1+p w)(1+w)}{w}\right)^{n} .
$$

Also, the $\mathcal{T}_{t_{i}, t_{i-1}}(x, y)$ from [4] now becomes

$$
\widetilde{F}_{0}\left(x-y, t_{i}-t_{i-1}\right)=\frac{1}{2 \pi \mathrm{i}} \oint_{\Gamma_{0,-1}} \mathrm{~d} w \frac{(1+p w)^{t_{i}-t_{i-1}}}{(1+w)^{x-y+1}} .
$$


The only step we have still to prove is that the space where we need to do the orthogonalization is actually $V_{n}=\operatorname{span}\left(1, x, \ldots, x^{n-1}\right)$. This follows from Lemma 12 below.

Lemma 12. We have the identity

$$
\sum_{x_{l}^{k}, 1 \leq l \leq k<n} \prod_{k=1}^{n-1} \operatorname{det}\left(\phi^{\sharp}\left(x_{i}^{k}, x_{j}^{k+1}\right)\right)_{1 \leq i, j \leq k+1}=C_{n} \prod_{1 \leq i<j \leq n}\left(x_{j}^{n}-x_{i}^{n}\right) .
$$

for some constant $C_{n} \neq 0$.

Proof of Lemma 12. The proof is made by induction. Assume that (4.12) is true for some $n$, which is the case for $n=2$. Then we prove that (4.12) holds for $n+1$. Consider

$$
\varphi_{k}(x, y)= \begin{cases}1-q, & x=y \\ 1, & y>x \\ 0, & y<x\end{cases}
$$

We will prove (4.12) for $\varphi$ instead of $\phi^{\#}$; the statements are clearly equivalent by shifts $x_{i}^{k} \mapsto x_{i}^{k}+k$. Set

$$
G_{k}^{n}(x)=\frac{1}{2 \pi \mathrm{i}} \oint_{|z| \gg 1} \mathrm{~d} z \frac{(q+(1-q) z)^{n-k}}{(z-1)^{n-k+1}} z^{x}
$$

Then, for $x \geq 0, G_{n}^{n}(x)=1$ and $G_{k}^{n}(x)$ is a polynomial of degree $n-k$ in $x$ (by evaluating the residue at $z=1$ ), while for $x<0, G_{k}^{n}(x)=0$ (because the residue at $\infty$ gives zero). Therefore

$$
\operatorname{det}\left(G_{k}^{n}\left(x_{j}^{n}\right)\right)_{1 \leq j, k \leq n}=C_{n} \prod_{1 \leq i<j \leq n}\left(x_{j}^{n}-x_{i}^{n}\right) .
$$

For the proof by induction, we apply Heine identity,

$$
\begin{aligned}
& \sum_{x_{1}, \ldots, x_{n}} \operatorname{det}\left(G_{k}^{n}\left(x_{j}\right)\right)_{1 \leq j, k \leq n} \operatorname{det}\left(\varphi_{n+1}\left(x_{i}, y_{j}\right)\right)_{1 \leq i, j \leq n+1} \\
= & n ! \operatorname{det}\left(\left(G_{k}^{n} * \varphi_{n+1}\right)\left(y_{j}\right)\right)_{1 \leq j, k \leq n+1} .
\end{aligned}
$$

The computation of the convolution leads to $\left(G_{k}^{n} * \varphi_{n+1}\right)(y)=G_{k}^{n+1}(y)$.

Theorem 11 holds for general fixed initial conditions. We want to apply it to the alternating initial condition. For that we first have to do the orthogonalization with the result given in the next lemma. 
Lemma 13. For initial conditions $y_{j}=-2 j, j=1, \ldots, n$, we have

$$
\Psi_{j}^{n, t}(x)=\frac{1}{2 \pi \mathrm{i}} \oint_{\Gamma_{0,-1}} \mathrm{~d} w \frac{w^{j}(1+p w)^{t-j}}{(1+w)^{x+2 n-j+1}},
$$

and

$$
\Phi_{j}^{n, t}(x)=\frac{1}{2 \pi \mathrm{i}} \oint_{\Gamma_{0}} \mathrm{~d} z \frac{\left(1+2 z+p z^{2}\right)(1+z)^{x+2 n-j-1}}{z^{j+1}(1+p z)^{t-j+1}},
$$

where, as before, $p=1-q$. In particular, $\Phi_{0}^{n, t}(x)=1$.

Proof of Lemma 13. The formula for $\Psi_{j}^{n}$ is just obtained by substituting the initial conditions into (4.4). Now we prove that the orthonormality relation (4.5) holds. For $k=0, \ldots, n-1$, the pole at $w=0$ in $\Psi_{k}^{n}$ is not present, and for $x<-2 n+k, \Psi_{k}^{n}(x)=0$ because the residue at -1 vanishes. Thus

$$
\begin{aligned}
& \sum_{x \in \mathbb{Z}} \Phi_{j}^{n, t}(x) \Psi_{k}^{n, t}(x) \\
= & \frac{1}{(2 \pi \mathrm{i})^{2}} \oint_{\Gamma_{0}} \mathrm{~d} z \oint_{\Gamma_{-1}} \mathrm{~d} w \frac{\left(1+2 z+p z^{2}\right)(1+z)^{2 n-j-1}}{z^{j+1}(1+p z)^{t-j+1}} \frac{w^{k}(1+p w)^{t-k}}{(1+w)^{2 n-k-1}} \\
& \times \sum_{x=-2 n+k}^{\infty}\left(\frac{1+z}{1+w}\right)^{x}
\end{aligned}
$$

where we have the constraint on the integration paths $|1+z|<|1+w|$. The last term (the sum) equals

$$
\left(\frac{1+z}{1+w}\right)^{-2 n+k} \frac{1+w}{w-z}
$$

Now the pole at $w=-1$ has disappeared and instead of it there is a simple pole at $w=z$. Thus, the integral over $w$ is just the residue at $w=z$, leading to

$$
\begin{aligned}
\sum_{x \in \mathbb{Z}} \Phi_{j}^{n, t}(x) \Psi_{k}^{n, t}(x) & =\frac{1}{2 \pi \mathrm{i}} \oint_{\Gamma_{0}} \mathrm{~d} z \frac{1+2 z+p z^{2}}{(1+p z)^{2}}\left(\frac{z(1+z)}{1+p z}\right)^{k-j-1} \\
& =\frac{1}{2 \pi \mathrm{i}} \int_{\Gamma_{0}} \mathrm{~d} u u^{k-j-1}=\delta_{j, k}
\end{aligned}
$$

where we used the change of variable $u=\frac{z(1+z)}{1+p z}$.

Lemma 13 together with Theorem 11 leads to the kernel for the alternating initial condition. 
Proposition 14. For $y_{j}=-2 j, j=1, \ldots, n$, the kernel $K$ in Theorem 11 is given by

$$
\begin{aligned}
K\left(\left(n_{1}, t_{1}\right), x_{1} ;\left(n_{2}, t_{2}\right), x_{2}\right)= & -\phi^{\left(\left(n_{1}, t_{1}\right),\left(n_{2}, t_{2}\right)\right)}\left(x_{1}, x_{2}\right) \mathbb{1}_{\left[\left(n_{1}, t_{1}\right) \prec\left(n_{2}, t_{2}\right)\right]} \\
& +\widetilde{K}\left(\left(n_{1}, t_{1}\right), x_{1} ;\left(n_{2}, t_{2}\right), x_{2}\right)
\end{aligned}
$$

where $\phi^{\left(\left(n_{1}, t_{1}\right),\left(n_{2}, t_{2}\right)\right)}\left(x_{1}, x_{2}\right)$ is given by (2.6) and

$$
\begin{aligned}
\widetilde{K}\left(\left(n_{1}, t_{1}\right), x_{1} ;\left(n_{2}, t_{2}\right), x_{2}\right)= & \frac{1}{(2 \pi \mathrm{i})^{2}} \oint_{\Gamma_{-1}} \mathrm{~d} w \oint_{\Gamma_{0}} \mathrm{~d} z \frac{w^{n_{1}}(1+p w)^{t_{1}-n_{1}+1}}{(1+w)^{x_{1}+n_{1}+1}} \\
& \times \frac{(1+z)^{x_{2}+n_{2}}\left(1+2 z+p z^{2}\right)}{z^{n_{2}}(1+p z)^{t_{2}-n_{2}+2}} \frac{1}{(w-z)\left(w+\frac{1+z}{1+p z}\right)} .
\end{aligned}
$$

Here $\Gamma_{0}\left(r e s p \Gamma_{-1}\right)$ is any simple loop, anticlockwise oriented, which includes the pole at $z=0$ (resp. $w=-1)$, satisfying $\left\{-\frac{1+z}{1+p z}, z \in \Gamma_{0}\right\} \subset \Gamma_{-1}$ and no point of $\Gamma_{0}$ lies inside $\Gamma_{-1}$.

Proof of Proposition 14. We substitute (4.17) and (4.18) in the kernel (4.2). Since $\Phi_{j}^{n, t}(x)=0$ for $j<0$, we can extend the sum over $k$ to $\infty$. We can take the sum inside the integrals if the integration paths satisfy $\left|\frac{1+p w}{w(1+w)} \frac{z(1+z)}{1+p z}\right|<1$. Then we compute the geometric series and obtain

$$
\begin{aligned}
\sum_{k=1}^{\infty} \Psi_{n_{1}-k}^{n_{1}, t_{1}}\left(x_{1}\right) \Phi_{n_{2}-k}^{n_{2}, t_{2}}\left(x_{2}\right)= & \frac{1}{(2 \pi \mathrm{i})^{2}} \oint_{\Gamma_{0,-1}} \mathrm{~d} w \oint_{\Gamma_{0}} \mathrm{~d} z \frac{w^{n_{1}}(1+p w)^{t_{1}-n_{1}+1}}{(1+w)^{x_{1}+n_{1}+1}} \\
& \times \frac{(1+z)^{x_{2}+n_{2}}\left(1+2 z+p z^{2}\right)}{z^{n_{2}}(1+p z)^{t_{2}-n_{2}+2}} \frac{1}{(w-z)\left(w+\frac{1+z}{1+p z}\right)} .
\end{aligned}
$$

At this point both simple poles $w=z$ and $w=-(1+z) /(1+p z)$ are inside the integration path $\Gamma_{0,-1}$, but the integrand does not have any pole anymore at $w=0$. Thus we will drop the 0 in $\Gamma_{0,-1}$. Separating the contribution from the pole at $w=z$ we get

$$
\begin{aligned}
\sum_{k=1}^{n_{2}} \Psi_{n_{1}-k}^{n_{1}, t_{1}}\left(x_{1}\right) \Phi_{n_{2}-k}^{n_{2}, t_{2}}\left(x_{2}\right) & =\widetilde{K}\left(\left(n_{1}, t_{1}\right), x_{1} ;\left(n_{2}, t_{2}\right), x_{2}\right) \\
& +\frac{1}{2 \pi \mathrm{i}} \oint_{\Gamma_{0}}\left(\frac{1+p z}{z}\right)^{n_{2}-n_{1}}(1+z)^{n_{2}+x_{2}-n_{1}-x_{1}-1}
\end{aligned}
$$

Moreover, we also have

$$
\begin{aligned}
\phi^{*\left(\left(n_{1}, t_{1}\right),\left(n_{2}, t_{2}\right)\right)}\left(x_{1}, x_{2}\right) & =\phi^{\left(\left(n_{1}, t_{1}\right),\left(n_{2}, t_{2}\right)\right)}\left(x_{1}, x_{2}\right) \\
& +\frac{1}{2 \pi \mathrm{i}} \oint_{\Gamma_{0}}\left(\frac{1+p z}{z}\right)^{n_{2}-n_{1}}(1+z)^{n_{2}+x_{2}-n_{1}-x_{1}-1} .
\end{aligned}
$$


Thus the last two terms of (4.25) and (4.26) cancel out, leading to (4.23).

With Proposition 14 we almost obtained Theorem 1, What remains to do is to focus far enough into the negative axis, where the influence of the finiteness of the number of particles is not present anymore. There the kernel is equal to the kernel for the initial conditions $y_{i}=-2 i, i \in \mathbb{Z}$.

Proof of Theorem 1. The kernel for the flat case is obtained by considering the region satisfying $x_{1}+n_{1}+1 \leq 0$ where the effect of the boundary in the TASEP is absent. Here the pole at $w=-1$ vanishes. Computing the residue at $w=-(1+z) /(1+p z)$ in Proposition 14 gives the kernel (2.5) up to a factor $(-1)^{n_{1}-n_{2}}$ which we cancel by a conjugation of the kernel.

\section{$5 \quad$ Proof of Theorem 3}

From Theorem 1 we have that $\mathbb{P}\left(x_{n}(t) \geq x\right)=\operatorname{det}\left(\mathbb{1}-\mathbb{1}_{(-\infty, x)} K \mathbb{1}_{(-\infty, x)}\right)$. We have such a situation but with $x=y-s T^{1 / 3}$. With this change of variable, we get $\mathbb{P}\left(x_{n}(t) \geq y-s T^{1 / 3}\right)=\operatorname{det}\left(\mathbb{1}-\mathbb{1}_{(s, \infty)} K_{T}^{\mathrm{resc}} \mathbb{1}_{(s, \infty)}\right)$ where $K_{T}^{\text {resc }}\left(\xi_{1}, \xi_{2}\right)=T^{1 / 3} K\left(x_{1}-\xi_{1} T^{1 / 3}, x_{2}-\xi_{2} T^{1 / 3}\right)$ (here we did not write explicitly the $(n, t)$ entries). Taking into account the scaling (2.10), we thus have to analyze the rescaled kernel

$$
\begin{aligned}
& K_{T}^{\mathrm{resc}}\left(u_{1}, \xi_{1} ; u_{2}, \xi_{2}\right) \\
= & T^{1 / 3} K\left(\left(n\left(u_{1}\right), t\left(u_{1}\right)\right), x\left(u_{1}\right)-\xi_{1} T^{1 / 3} ;\left(n\left(u_{2}\right), t\left(u_{2}\right)\right), x\left(u_{2}\right)-\xi_{2} T^{1 / 3}\right)
\end{aligned}
$$

with $x(u)=-2 n(u)+\mathbf{v} t(u), \mathbf{v}=1-\sqrt{q}$. In particular, we have to prove that, for $u_{1}, u_{2}$ fixed, $K_{T}^{\text {resc }}$ (or a conjugate kernel of it) converges to the kernel $\kappa_{\mathrm{v}}^{-1} K_{\mathcal{A}_{1}}\left(\kappa_{\mathrm{h}}^{-1} u_{1}, \kappa_{\mathrm{v}}^{-1} \xi_{1}, \kappa_{\mathrm{h}}^{-1} u_{2}, \kappa_{\mathrm{v}}^{-1} \xi_{2}\right)$ uniformly on bounded sets and have enough control (bounds) on the decay of $K^{\text {resc }}$ in the variables $\xi_{1}, \xi_{2}$ such that also the Fredholm determinant converges.

In order to have a proper limit of the kernel as $T \rightarrow \infty$, we have to consider the conjugate kernel $K_{T}^{\text {conj }}$ given by

$$
K_{T}^{\mathrm{conj}}\left(u_{1}, \xi_{1} ; u_{2}, \xi_{2}\right)=K_{T}^{\mathrm{resc}}\left(u_{1}, \xi_{1} ; u_{2}, \xi_{2}\right)\left(\frac{\sqrt{q}}{1+\sqrt{q}}\right)^{x_{1}-x_{2}} q^{n_{1}-n_{2}} q^{-\left(t_{1}-t_{2}\right) / 2} .
$$

The new kernel does not change the determinantal measure, being just a conjugation of the old one. So, in the following we will determine the limit of (5.2) as $T \rightarrow \infty$. 
Proposition 15 (Uniform convergence on compact sets). For $u_{1}, u_{2}$ fixed, according to (2.10), set

$$
\begin{aligned}
& x_{i}=\left[-2 n\left(u_{i}\right)+\mathbf{v} t\left(u_{i}\right)-\xi_{i} T^{1 / 3}\right], \\
& n_{i}=n\left(u_{i}\right), \quad t_{i}=t\left(u_{i}\right) .
\end{aligned}
$$

Then, for any fixed $L>0$, we have

$$
\lim _{T \rightarrow \infty} K_{T}^{\text {conj }}\left(n_{1}, x_{1} ; n_{2}, x_{2}\right) T^{1 / 3}=\kappa_{\mathrm{v}}^{-1} K_{\mathcal{A}_{1}}\left(\kappa_{\mathrm{h}}^{-1} u_{1}, \kappa_{\mathrm{v}}^{-1} \xi_{1}, \kappa_{\mathrm{h}}^{-1} u_{2}, \kappa_{\mathrm{v}}^{-1} \xi_{2}\right)
$$

uniformly for $\left(\xi_{1}, \xi_{2}\right) \in[-L, L]^{2}$, with the kernel $K_{\mathcal{A}_{1}}$ given by (2.7) and the constants $\kappa_{\mathrm{v}}$ and $\kappa_{\mathrm{h}}$ given by (2.13).

Proof of Proposition 15. First we consider the first term in (2.4). We thus consider (2.6) with the above replacements and conjugation. This term has to be considered only for $u_{2}>u_{1}$. The change of variable $w=-1+\sqrt{q} z$ leads then to

$$
\frac{T^{1 / 3}}{2 \pi \mathrm{i}} \oint_{\Gamma_{0}} \frac{\mathrm{d} z}{z} e^{T^{2 / 3}\left(g_{0}(z)-g_{0}\left(z_{c}\right)\right)+T^{1 / 3}\left(g_{1}(z)-g_{1}\left(z_{c}\right)\right)}
$$

with $z_{c}=(1+\sqrt{q})^{-1}$ and

$$
\begin{aligned}
g_{0}(z) & =\left(u_{2}-u_{1}\right)\left(\pi^{\prime}(\theta)+1\right)(\ln (\sqrt{q}+(1-q) z)-(1-\sqrt{q}) \ln (z)) \\
& +\left(u_{2}-u_{1}\right)\left(1-\pi^{\prime}(\theta)\right) \ln \left(\frac{\sqrt{q}+(1-q) z}{z(1-\sqrt{q} z)}\right), \\
g_{1}(z) & =-\left(u_{2}^{2}-u_{1}^{2}\right) \frac{1}{2} \pi^{\prime \prime}(\theta)(\sqrt{q} \ln (z)+\ln (1-\sqrt{q} z))-\left(\xi_{2}-\xi_{1}\right) \ln (z) .
\end{aligned}
$$

The function $g_{0}$ has a critical point at $z=z_{c}$. The series expansions around $z=z_{c}$ are

$$
\begin{aligned}
& g_{0}(z)=g_{0}\left(z_{c}\right)+\left(u_{2}-u_{1}\right) \kappa_{1}\left(z-z_{c}\right)^{2}+\mathcal{O}\left(\left(z-z_{c}\right)^{3}\right), \\
& g_{1}(z)=g_{1}\left(z_{c}\right)-\left(\xi_{2}-\xi_{1}\right)(1+\sqrt{q})\left(z-z_{c}\right)+\mathcal{O}\left(\left(z-z_{c}\right)^{2}\right),
\end{aligned}
$$

where

$$
\kappa_{1}=\sqrt{q}(1+\sqrt{q})^{2}\left[\left(\pi^{\prime}(\theta)+1\right) \frac{1-\sqrt{q}}{2}+1-\pi^{\prime}(\theta)\right] .
$$

To prove convergence of $(5.6)$ we have to show that the contribution coming around the critical point dominates in the $T \rightarrow \infty$ limit. We do it by finding a steep descent path ${ }^{2}$ for $g_{0}$ passing by $z=z_{c}$. Consider the path

\footnotetext{
${ }^{2}$ For an integral $I=\int_{\gamma} \mathrm{d} z e^{t f(z)}$, we say that $\gamma$ is a steep descent path if $(1) \operatorname{Re}(f(z))$ is maximal at some $z_{0} \in \gamma: \operatorname{Re}(f(z))<\operatorname{Re}\left(f\left(z_{0}\right)\right)$ for $z \in \gamma \backslash\left\{z_{0}\right\}$ and $(2) \operatorname{Re}(f(z))$ is monotone along $\gamma \backslash\left\{z_{0}\right\}$ except, if $\gamma$ is closed, at a single point where $\operatorname{Re}(f)$ is minimal.
} 
$\Gamma_{0}=\left\{\rho e^{i \phi}, \phi \in[-\pi, \pi)\right\}$. Then, on $\Gamma_{0}, \frac{\mathrm{d}}{\mathrm{d} \phi} \operatorname{Re}(\ln (z))=0$,

$$
\frac{\mathrm{d}}{\mathrm{d} \phi} \operatorname{Re}(\ln (\sqrt{q}+(1-q) z))=-\frac{\sqrt{q}(1-q) \rho \sin (\phi)}{|\sqrt{q}+(1-q) z|^{2}},
$$

and

$$
\frac{\mathrm{d}}{\mathrm{d} \phi} \operatorname{Re}(-\ln (1-\sqrt{q} z))=-\frac{\sqrt{q} \rho \sin (\phi)}{|1-\sqrt{q} z|^{2}} .
$$

Thus $\Gamma_{0}$ is a steep descent path for $g_{0}$. Now we set $\rho=z_{c}$. Then, the real part of $g_{0}(z)$ is maximal at $z=z_{c}$ and strictly less then $g\left(z_{c}\right)$ for all other points on $\Gamma_{0}$. Therefore, we can restrict the integration from $\Gamma_{0}$ to $\Gamma_{0}^{\delta}=\left\{z \in \Gamma_{0}|| z-z_{c} \mid \leq \delta\right\}$. For $\delta$ small, the error made is just of order $\mathcal{O}\left(e^{-c T^{2 / 3}}\right)$ with $c>0\left(c \sim \delta^{2}\right.$ as $\left.\delta \ll 1\right)$. In the integral over $\Gamma_{0}^{\delta}$ we can use (5.8) to get

$$
\begin{aligned}
& \frac{(1+\sqrt{q}) T^{1 / 3}}{2 \pi \mathrm{i}} \oint_{\Gamma_{0}^{\delta}} \mathrm{d} z e^{T^{2 / 3}\left(u_{2}-u_{1}\right) \kappa_{1}\left(z-z_{c}\right)^{2}-T^{1 / 3}\left(\xi_{2}-\xi_{1}\right)(1+\sqrt{q})\left(z-z_{c}\right)} \\
\times & e^{\mathcal{O}\left(T^{2 / 3}\left(z-z_{c}\right)^{3}, T^{1 / 3}\left(z-z_{c}\right)^{2},\left(z-z_{c}\right)\right)} .
\end{aligned}
$$

We use $\left|e^{x}-1\right| \leq|x| e^{|x|}$ to control the difference between (5.12) and the same expression without the error terms. By taking $\delta$ small enough and the change of variable $\left(z-z_{c}\right) T^{1 / 3}=W$, we obtain that this difference is just of order $\mathcal{O}\left(T^{-1 / 3}\right)$, uniformly for $\xi_{1}, \xi_{2}$ in a bounded set. At this point we remain with (5.12) without the error terms. We extend the integration path to $z_{c}+\mathrm{i} \mathbb{R}$ and this, as above, gives an error of order $\mathcal{O}\left(e^{-c T^{2 / 3}}\right)$. Thus we have

$$
\begin{aligned}
(\underline{5.6}) & =\mathcal{O}\left(e^{-c T^{2 / 3}}, T^{-1 / 3}\right) \\
& +\frac{(1+\sqrt{q}) T^{1 / 3}}{2 \pi \mathrm{i}} \oint_{z_{c}+\mathrm{i} \mathbb{R}} \mathrm{d} z e^{T^{2 / 3}\left(u_{2}-u_{1}\right) \kappa_{1}\left(z-z_{c}\right)^{2}-T^{1 / 3}\left(\xi_{2}-\xi_{1}\right)(1+\sqrt{q})\left(z-z_{c}\right)} .
\end{aligned}
$$

Therefore, uniformly for $\xi_{1}, \xi_{2}$ in bounded sets,

$$
\lim _{T \rightarrow \infty}(\underline{5.6})=\frac{1}{\sqrt{4 \pi\left(u_{2}-u_{1}\right) \alpha}} \exp \left(-\frac{\left(\xi_{2}-\xi_{1}\right)^{2}}{4\left(u_{2}-u_{1}\right) \alpha^{2}}\right)
$$

with $\alpha^{2}=\kappa_{1} /(1+\sqrt{q})^{2}=\kappa_{\mathrm{v}}^{2} / \kappa_{\mathrm{h}}$.

Now we have to consider the second term in (2.4). Notice that this time the restriction $u_{2}>u_{1}$ does not apply. Set $z_{c}=-1 /(1+\sqrt{q})$. Then

$$
\widetilde{K}_{T}^{\text {conj }}\left(u_{1}, \xi_{1} ; u_{2}, \xi_{2}\right)=\frac{-T^{1 / 3}}{2 \pi \mathrm{i}} \oint_{\Gamma_{0}} \mathrm{~d} z \frac{e^{T f_{0}(z)+T^{2 / 3} f_{1}(z)+T^{1 / 3} f_{2}(z)+f_{3}(z)}}{e^{T^{2 / 3} f_{1}\left(z_{c}\right)+T^{1 / 3} f_{2}\left(z_{c}\right)}}
$$


with

$$
\begin{aligned}
& f_{0}(z)=(\pi(\theta)+\theta)\left[(1-\sqrt{q}) \ln \left(\frac{1+z}{-z}\right)-(1+\sqrt{q}) \ln (1+(1-q) z)+\sqrt{q} \ln (q)\right] \\
& \begin{aligned}
& f_{1}(z)=\left(\pi^{\prime}(\theta)+1\right) {\left[u_{1}((1-\sqrt{q}) \ln (-z)+\sqrt{q} \ln ((1+(1-q) z) / q))\right.} \\
&\left.-u_{2}((1-\sqrt{q}) \ln (1+z)-\ln (1+(1-q) z))\right] \\
&+\left(1-\pi^{\prime}(\theta)\right)\left(u_{1}-u_{2}\right) \ln \left(\frac{(1+z)(-z)}{1+(1-q) z}\right) \\
&\left.\quad-u_{2}^{2}(\sqrt{q} \ln (1+z)+\ln (-z))\right] \\
& f_{2}(z)=\frac{\pi^{\prime \prime}(\theta)}{2}\left[u_{1}^{2}(\ln (1+z)+\sqrt{q} \ln (-q z)-(1+\sqrt{q}) \ln (1+(1-q) z))\right. \\
& \quad+\xi_{1} \ln (-q z /(1+(1-q) z))-\xi_{2} \ln (1+z) \\
& f_{3}(z)=-\ln (-z(1+(1-q) z)) .
\end{aligned}
\end{aligned}
$$

The function $f_{0}$ has a double critical point at $z=z_{c}$ and the series expansions around $z=z_{c}$ of the $f_{i}$ 's are given by

$$
\begin{aligned}
& f_{0}(z)=\frac{1}{3} \kappa_{2}\left(z-z_{c}\right)^{3}+\mathcal{O}\left(\left(z-z_{c}\right)^{4}\right), \\
& f_{1}(z)=f_{1}\left(z_{c}\right)+\frac{\kappa_{1}}{q}\left(u_{2}-u_{1}\right)\left(z-z_{c}\right)^{2}+\mathcal{O}\left(\left(z-z_{c}\right)^{3}\right), \\
& f_{2}(z)=f_{2}\left(z_{c}\right)-\left(\xi_{1}+\xi_{2}\right) \frac{1+\sqrt{q}}{\sqrt{q}}\left(z-z_{c}\right)+\mathcal{O}\left(\left(z-z_{c}\right)^{2}\right), \\
& f_{3}(z)=\ln ((1+\sqrt{q}) / \sqrt{q})+\mathcal{O}\left(\left(z-z_{c}\right)\right),
\end{aligned}
$$

with $\kappa_{1}$ given in (5.9) and

$$
\kappa_{2}=\frac{(\pi(\theta)+\theta)(1-q)(1+\sqrt{q})^{3}}{q} .
$$

The leading contribution in the $T \rightarrow \infty$ limit will come from the region around the double critical point. The first step is to choose for $\gamma_{0}$ a steep descent path for $f_{0}$. First we consider $\gamma_{0}=\left\{-\rho e^{\mathrm{i} \phi}, \phi \in[-\pi, \pi)\right\}$, $\rho \in(0,1 /(1+\sqrt{q})]$. The only part in $\operatorname{Re}\left(f_{0}(z)\right)$ which is not constant along $\gamma_{0}$ is the term $(\pi(\theta)+\theta)(1-\sqrt{q})$ multiplied by $A(z)=\ln |1+z|-a \ln |1+(1-q) z|$, $a=(1+\sqrt{q}) /(1-\sqrt{q})$. Simple computations lead then to

$$
\frac{\mathrm{d}}{\mathrm{d} \phi} A(z)=-\frac{\sin (\phi) \rho \sqrt{q}\left(2+\sqrt{q}-4 \rho(1+\sqrt{q}) \cos (\phi)+\rho^{2}(2-\sqrt{q})(1+\sqrt{q})^{2}\right)}{|1+z|^{2}|1+(1-q) z|^{2}} .
$$


This expression is strictly less than zero along $\gamma_{0}$ except at $\phi=0,-\pi$, provided that the last term is strictly positive for $\phi \neq 0,-\pi$. This is easy to check because the last term reaches his minimum at $\cos (\phi)=-1$. Solving a second degree equations, we get that on $\rho \in(0,1 /(1+\sqrt{q}))$ it is strictly positive and at $\rho=1 /(1+\sqrt{q})$ is zero. Thus, the path $\gamma_{0}$ is steep descent for $f_{0}$.

But close to the critical point, the steepest descent path leaves with an angle $\pm \pi / 3$. Therefore, consider for a moment $\gamma_{1}=\left\{z=z_{c}+e^{-\mathrm{i} \pi \operatorname{sgn}(x) / 3}|x|, x \in\right.$ $\mathbb{R}\}$. By symmetry we can restrict the next computations to $x \geq 0$. We have to see that $B(z)=\ln |1+z|-\ln |z|-a \ln |1+(1-q) z|$ is maximum at $x=0$ and decreasing for $x>0$. We have

$$
\begin{aligned}
& \frac{\mathrm{d}}{\mathrm{d} x} B(z)=-\frac{x^{2}}{2|1+z|^{2}|z|^{2}|1+(1-q) z|^{2}} \\
& \times \quad\left(2 q+2(1-q) \sqrt{q} x-(1-3 \sqrt{q}+q)(1+\sqrt{q})^{2} x^{2}+2(1-q)(1+\sqrt{q})^{3} x^{3}\right) .
\end{aligned}
$$

The term in the second line is always positive for all $x \geq 0$. To see this, remark that it is a polynomial of third degree which goes to $\infty$ as $x \rightarrow \infty$ and at $x=0$ is already positive and has positive slope. Therefore one just computes its stationary points and, if reals, takes the right-most one. There, the term under consideration turns out to be positive, which concludes the argument. Consequently, $\gamma_{1}$ is also a steep descent path.

We choose a steep descent path $\Gamma_{0}$ as follows. We follow $\gamma_{1}$ starting from the critical point until we intersect it with $\gamma_{0}$, and then we follow $\gamma_{0}$. Since $\Gamma_{0}$ is steep descent for $f_{0}$, we can integrate only on $\Gamma_{0}^{\delta}=\left\{z \in \Gamma_{0}|| z-z_{c} \mid \leq \delta\right\}$. The error made by this cut is just of order $\mathcal{O}\left(e^{-c T}\right)$ for some $c=c(\delta)>0$ (with $c \sim \delta^{3}$ as $\delta \rightarrow 0$ ). Around the critical point we use the series expansions (5.18). Thus we have

$$
\begin{aligned}
& \widetilde{K}_{T}^{\text {conj }}\left(u_{1}, \xi_{1} ; u_{2}, \xi_{2}\right)=\mathcal{O}\left(e^{-c T}\right) \\
& +\frac{-T^{1 / 3}}{2 \pi \mathrm{i}} \int_{\Gamma_{0}^{\delta}} \mathrm{d} z \frac{1+\sqrt{q}}{\sqrt{q}} e^{\mathcal{O}\left(\left(z-z_{c}\right)^{4} T,\left(z-z_{c}\right)^{3} T^{2 / 3},\left(z-z_{c}\right)^{2} T^{1 / 3},\left(z-z_{c}\right)\right)} \\
& \times e^{\frac{1}{3} \kappa_{2}\left(z-z_{c}\right)^{3} T+\frac{\kappa_{1}}{q}\left(u_{2}-u_{1}\right)\left(z-z_{c}\right)^{2} T^{2 / 3}-\left(\xi_{1}+\xi_{2}\right) \frac{1+\sqrt{q}}{\sqrt{q}}\left(z-z_{c}\right) T^{1 / 3}}
\end{aligned}
$$

We want to cancel the error terms. The difference between (5.22) and the same expression without the error terms is bounded using $\left|e^{x}-1\right| \leq e^{|x|}|x|$, applied to $x=\mathcal{O}(\cdots)$. Then, this error term becomes

$$
\begin{aligned}
& \mid \frac{T^{1 / 3}}{2 \pi \mathrm{i}} \int_{\Gamma_{0}^{\delta}} \mathrm{d} z \frac{1+\sqrt{q}}{\sqrt{q}} \mathcal{O}\left(\left(z-z_{c}\right)^{4} T,\left(z-z_{c}\right)^{3} T^{2 / 3},\left(z-z_{c}\right)^{2} T^{1 / 3},\left(z-z_{c}\right)\right) \\
& \times e^{c_{1} \frac{1}{3} \kappa_{2}\left(z-z_{c}\right)^{3} T+c_{2} \frac{\kappa_{1}}{q}\left(u_{2}-u_{1}\right)\left(z-z_{c}\right)^{2} T^{2 / 3}-c_{3}\left(\xi_{1}+\xi_{2}\right) \frac{1+\sqrt{q}}{\sqrt{q}}\left(z-z_{c}\right) T^{1 / 3}} \mid
\end{aligned}
$$


for some $c_{1}, c_{2}, c_{3}$ depending on $\delta$. As $\delta \rightarrow 0$, the $c_{i} \rightarrow 1$. Thus, for $\delta$ small enough, we have $c_{1}>0$. By the change of variable $\left(z-z_{c}\right) T^{1 / 3}=W$ we obtain that (5.23) is just of order $\mathcal{O}\left(T^{-1 / 3}\right)$. Thus we have

$$
\begin{aligned}
& \widetilde{K}_{T}^{\text {conj }}\left(u_{1}, \xi_{1} ; u_{2}, \xi_{2}\right)=\mathcal{O}\left(e^{-c T}, T^{-1 / 3}\right) \\
& +\frac{-T^{1 / 3}}{2 \pi \mathrm{i}} \int_{\Gamma_{0}^{\delta}} \mathrm{d} z \frac{1+\sqrt{q}}{\sqrt{q}} e^{\frac{1}{3} \kappa_{2}\left(z-z_{c}\right)^{3} T+\frac{\kappa_{1}}{q}\left(u_{2}-u_{1}\right)\left(z-z_{c}\right)^{2} T^{2 / 3}-\left(\xi_{1}+\xi_{2}\right) \frac{1+\sqrt{q}}{\sqrt{q}}\left(z-z_{c}\right) T^{1 / 3}} .
\end{aligned}
$$

The extension of the path $\Gamma_{0}^{\delta}$ to a path going from $e^{\mathrm{i} \pi / 3} \infty$ to $e^{-\mathrm{i} \pi / 3} \infty$ accounts into an error $\mathcal{O}\left(e^{-c T}\right)$ only. We do the change of variable $Z=\kappa_{2}^{1 / 3} T^{1 / 3}\left(z-z_{c}\right)$ and we define

$$
\kappa_{\mathrm{v}}=\frac{\kappa_{2}^{1 / 3} \sqrt{q}}{1+\sqrt{q}}, \quad \kappa_{\mathrm{h}}=\frac{\kappa_{2}^{2 / 3} q}{\kappa_{1}} .
$$

Then,

$$
\lim _{T \rightarrow \infty} \widetilde{K}_{T}^{\text {conj }}\left(u_{1}, \xi_{1} ; u_{2}, \xi_{2}\right)=\kappa_{\mathrm{v}}^{-1} \frac{-1}{2 \pi \mathrm{i}} \int_{\gamma_{\infty}} \mathrm{d} Z e^{\frac{1}{3} Z^{3}+\left(u_{2}-u_{1}\right) Z^{2} \kappa_{\mathrm{h}}^{-1}-\left(\xi_{1}+\xi_{2}\right) Z \kappa_{\mathrm{v}}^{-1}}
$$

where $\gamma_{\infty}$ is any path going from $e^{\mathrm{i} \pi / 3} \infty$ to $e^{-\mathrm{i} \pi / 3} \infty$. The proof ends by using the Airy function representation (A.5).

Proposition 16 (Bound for the diffusion term of the kernel). Let $n_{i}, t_{i}$, and $x_{i}$ be defined as in Proposition 15. Then, for $u_{2}-u_{1}>0$ fixed and for any $\xi_{1}, \xi_{2} \in \mathbb{R}$, the bound

$$
\left|\phi^{\left(\left(n_{1}, t_{1}\right),\left(n_{2}, t_{2}\right)\right)}\left(x_{1}, x_{2}\right) T^{1 / 3}\left(\frac{\sqrt{q}}{1+\sqrt{q}}\right)^{x_{1}-x_{2}} q^{n_{1}-n_{2}} q^{-\left(t_{1}-t_{2}\right) / 2}\right| \leq \text { const } e^{-\left|\xi_{1}-\xi_{2}\right|}
$$

holds for $T$ large enough and const independent of $T$.

Proof of Proposition 16. We start with (5.6). The difference now is that the contribution coming from large $\left|\xi_{1}-\xi_{2}\right|$ can be of the same order as the one from $g_{0}(z)$. We consider as path $\Gamma_{0}=\left\{\rho e^{\mathrm{i} \phi}, \phi \in[-\pi, \pi)\right\}$.

The difference is that now we choose $\rho$ as follows. For an $\varepsilon$ with $0<\varepsilon \ll 1$ and $z_{c}=1 /(1+\sqrt{q})$,

$$
\rho= \begin{cases}z_{c}+\frac{\left(\xi_{2}-\xi_{1}\right) T^{-1 / 3}}{\left(u_{2}-u_{1}\right) \kappa_{1}}, & \text { if }\left|\xi_{2}-\xi_{1}\right| \leq \varepsilon T^{1 / 3}, \\ z_{c}+\frac{\varepsilon}{\left(u_{2}-u_{1}\right) \kappa_{1}}, & \text { if } \xi_{2}-\xi_{1} \geq \varepsilon T^{1 / 3}, \\ z_{c}-\frac{\varepsilon}{\left(u_{2}-u_{1}\right) \kappa_{1}}, & \text { if } \xi_{2}-\xi_{1} \leq-\varepsilon T^{1 / 3} .\end{cases}
$$

By (5.10) and (5.11), $\Gamma_{0}$ is a steep descent path for $g_{0}(z)$ plus the term proportional to $\xi_{1}-\xi_{2}$ in $g_{1}(z)$. So, integrating on $\Gamma_{0}^{\delta}=\left\{z=\rho e^{\mathrm{i} \phi}, \phi \in\right.$ 
$(-\delta, \delta)\}$ instead of $\Gamma_{0}$ we do only an error of order $\mathcal{O}\left(e^{-c T^{2 / 3}}\right)$ times the value at $\phi=0$, for some $c>0$. Thus

$$
\begin{aligned}
\text { LHS of (5.27) } & =e^{T^{2 / 3}\left(g_{0}(\rho)-g_{0}\left(z_{c}\right)\right)+T^{1 / 3}\left(g_{1}(\rho)-g_{1}\left(z_{c}\right)\right)} \\
& \times\left(\mathcal{O}\left(e^{-c T^{2 / 3}}\right)+\frac{T^{1 / 3}}{2 \pi \mathrm{i}} \int_{\Gamma_{0}^{\delta}} \frac{\mathrm{d} z}{z} e^{T^{2 / 3}\left(g_{0}(z)-g_{0}(\rho)\right)+T^{1 / 3}\left(g_{1}(z)-g_{1}(\rho)\right)}\right) .
\end{aligned}
$$

On $\Gamma_{0}^{\delta}$, the $\xi_{i}$-dependent term in $\operatorname{Re}\left(g_{1}(z)-g_{1}(\rho)\right)$ is equal to zero. With the same procedure as in Proposition 15 one shows that the integral is bounded by a constant, uniformly in $T$.

It remains to estimate the first factor in (5.29). With our choice (5.28), we need just series expansions of $g_{0}$ and $g_{1}$ around $\rho$. Namely, by (5.8)

$$
\begin{aligned}
T^{2 / 3}\left(g_{0}(\rho)-g_{0}\left(z_{c}\right)\right) & =\left(u_{2}-u_{1}\right) \kappa_{1}\left(\rho-z_{c}\right)^{2} T^{2 / 3}\left(1+\mathcal{O}\left(\rho-z_{c}\right)\right) \\
T^{1 / 3}\left(g_{1}(\rho)-g_{1}\left(z_{c}\right)\right) & =\left(\xi_{1}-\xi_{2}\right)(1+\sqrt{q})\left(\rho-z_{c}\right) T^{1 / 3}\left(1+\mathcal{O}\left(\rho-z_{c}\right)\right) \\
& +\mathcal{O}\left(\left(\rho-z_{c}\right)^{2}\right) T^{1 / 3}
\end{aligned}
$$

First consider the case $\left|\xi_{2}-\xi_{1}\right| \leq \varepsilon T^{1 / 3}$. We replace $\rho$ given in (5.28) into (5.30) and get that the sum of the two contributions in (5.30) writes

$$
-\frac{\sqrt{q}\left(\xi_{2}-\xi_{1}\right)^{2}}{\left(u_{2}-u_{1}\right) \kappa_{1}}\left(1+\mathcal{O}(\varepsilon)+\mathcal{O}\left(T^{-1 / 3}\right)\right)
$$

$\mathcal{O}(\varepsilon)$ comes from $\mathcal{O}\left(\rho-z_{c}\right)$, while the $\mathcal{O}\left(T^{-1 / 3}\right)$ from $\mathcal{O}\left(\left(\rho-z_{c}\right)^{2}\right)$. Then, by taking $\varepsilon$ small enough and $T$ large enough, we get

$$
\text { (5.31) } \leq-\left|\xi_{2}-\xi_{1}\right|+\text { const. }
$$

In the case, $\xi_{2}-\xi_{1}>\varepsilon T^{1 / 3}$, we also replace the appropriate $\rho$ given in (5.28) into (5.30). We explicitly use the bound $\varepsilon T^{1 / 3}<\xi_{2}-\xi_{1}$ to bound $\mathcal{O}\left(\left(\rho-z_{c}\right)^{2}\right) \leq\left(\xi_{2}-\xi_{1}\right) T^{-1 / 3} \varepsilon$. Then, we obtain the following bound for the sum of the two contributions in (5.30),

$$
\left|\xi_{2}-\xi_{1}\right| \varepsilon T^{1 / 3}\left(\mathcal{O}\left(T^{-1 / 3}\right)-\frac{\sqrt{q}}{\left(u_{2}-u_{1}\right) \kappa_{1}}(1+\mathcal{O}(\varepsilon))\right) \leq-\left|\xi_{2}-\xi_{1}\right|
$$

by taking a fixed $\varepsilon$ small enough and then $T$ large enough. Finally, for $\xi_{2}-\xi_{1}<\varepsilon T^{1 / 3}$, the same result holds in a similar way.

Proposition 17 (Bound for the main term of the kernel). Let $n_{i}, t_{i}$, and $x_{i}$ be defined as in Proposition 15. Let $L>0$ fixed. Then, for given $u_{1}, u_{2}$ and $\xi_{1}, \xi_{2} \geq-L$, the bound

$$
\left|\widetilde{K}_{T}^{c o n j}\left(u_{1}, \xi_{1} ; u_{2}, \xi_{2}\right)\right| \leq \text { const } e^{-\left(\xi_{1}+\xi_{2}\right)}
$$

holds for $T$ large enough and const independent of $T$. 
Proof of Proposition 17. For $\xi_{1}, \xi_{2} \in[-L, L]$ it is the content of Proposition [15. Thus we consider $\xi_{1}, \xi_{2} \in[-L, \infty)^{2} \backslash[-L, L]^{2}$. Define $\tilde{\xi}_{i}=$ $\left(\xi_{i}+2 L\right) T^{-2 / 3}>0$. Then we consider a slight modification of (5.15), namely

$$
\widetilde{K}_{T}^{\text {conj }}\left(u_{1}, \xi_{1} ; u_{2}, \xi_{2}\right)=\frac{-T^{1 / 3}}{2 \pi \mathrm{i}} \oint_{\Gamma_{0}} \mathrm{~d} z \frac{e^{T \tilde{f}_{0}(z)+T^{2 / 3}} f_{1}(z)+T^{1 / 3} \tilde{f}_{2}(z)+f_{3}(z)}{e^{T \tilde{f}_{0}\left(z_{c}\right)+T^{2 / 3} f_{1}\left(z_{c}\right)+T^{1 / 3} \tilde{f}_{2}\left(z_{c}\right)}}
$$

with $f_{1}(z)$ and $f_{3}(z)$ as in (5.16)-(5.17), $\tilde{f}_{2}(z)$ as $f_{2}(z)$ in (5.17) but with $\xi_{1}$ and $\xi_{2}$ replaced by $-2 L$, and finally $\tilde{f}_{0}(z)$ is set to be equal to $f_{0}(z)$ in (5.16) plus the term

$$
-\tilde{\xi}_{1} \ln ((1+(1-q) z) /(-q z))-\tilde{\xi}_{2} \ln (1+z) .
$$

We also chose $\Gamma_{0}=\left\{-\rho e^{\mathrm{i} \phi}, \phi \in[-\pi, \pi)\right\}$. In the proof of Proposition 15] we already proved that $\Gamma_{0}$ is a steep descent path for $f_{0}$ for the values $\rho \in\left(0, z_{c}\right]$. Also, since $\tilde{\xi}_{i}>0, \operatorname{Re}((\underline{5.36}))$ is also decreasing while $|\phi|$ is increasing. The precise choice of $\rho$ is

$$
\rho= \begin{cases}-z_{c}-\left(\left(\tilde{\xi}_{1}+\tilde{\xi}_{2}\right) / \kappa_{2}\right)^{1 / 2}, & \text { if }\left|\tilde{\xi}_{1}+\tilde{\xi}_{2}\right| \leq \varepsilon \\ -z_{c}-\left(\varepsilon / \kappa_{2}\right)^{1 / 2}, & \text { if }\left|\tilde{\xi}_{1}+\tilde{\xi}_{2}\right| \geq \varepsilon\end{cases}
$$

for some small $\varepsilon>0$ which can be chosen later. Let us define

$$
Q(\rho)=e^{\operatorname{Re}\left(T\left(\tilde{f}_{0}(-\rho)-\tilde{f}_{0}\left(z_{c}\right)\right)+T^{2 / 3}\left(f_{1}(-\rho)-f_{1}\left(z_{c}\right)\right)+T^{1 / 3}\left(\tilde{f}_{2}(-\rho)-\tilde{f}_{2}\left(z_{c}\right)\right)\right)} .
$$

Then, since $\Gamma_{0}$ is a steep descent path for $\tilde{f}_{0}$,

$$
(\underline{5.35})=Q(\rho) \mathcal{O}\left(e^{-c T}\right)+Q(\rho) \frac{-T^{1 / 3}}{2 \pi \mathrm{i}} \int_{\Gamma_{0}^{\delta}} \mathrm{d} z \frac{e^{T \tilde{f}_{0}(z)+T^{2 / 3} f_{1}(z)+T^{1 / 3} \tilde{f}_{2}(z)+f_{3}(z)}}{e^{T \tilde{f}_{0}(\rho)+T^{2 / 3} f_{1}(\rho)+T^{1 / 3} \tilde{f}_{2}(\rho)}},
$$

where $\Gamma_{0}^{\delta}=\left\{-\rho e^{\mathrm{i} \phi}, \phi \in(-\delta, \delta)\right\}$, for a small $\delta>0$. The expansion around $\phi=0$ leads to

$$
\operatorname{Re}\left(\tilde{f}_{0}\left(-\rho e^{\mathrm{i} \phi}\right)-\tilde{f}_{0}(-\rho)\right)=-\gamma_{1} \phi^{2}(1+\mathcal{O}(\phi))
$$

with

$$
\begin{aligned}
\gamma_{1} & =\frac{(1-\rho(1+\sqrt{q}) \rho \sqrt{q}(1-\sqrt{q})(\rho q+(1-\rho)(2+\sqrt{q}))}{2(1-\rho)^{2}(1-\rho(1-q))^{2}} \\
& +\frac{\rho}{2}\left(\frac{\tilde{\xi}_{2}}{(1-\rho)^{2}}+\frac{\tilde{\xi}_{1}(1-q)}{(1-\rho(1-\rho))^{2}}\right)
\end{aligned}
$$

which is strictly positive for $\rho$ chosen as in (5.37). Also, $\operatorname{Re}\left(f_{1}\left(-\rho e^{\mathrm{i} \phi}\right)-\right.$ $\left.f_{1}(-\rho)\right)=\gamma_{2} \phi^{2}(1+\mathcal{O}(\phi))$ for some bounded $\gamma_{2}$ (we do not write it down 
explicitly since the precise formula is not relevant). Therefore, the last term in (5.39) is bounded by

$$
\text { const } Q(\rho) T^{1 / 3} \int_{-\delta}^{\delta} \mathrm{d} \phi e^{-\gamma \phi^{2} T(1+\mathcal{O}(\phi))\left(1+\mathcal{O}\left(T^{-1 / 3}\right)\right)}
$$

with $\gamma=\gamma_{1}+\gamma_{2} T^{-1 / 3}$. By choosing $\delta$ small enough and independent of $T$, and then $T$ large enough, the error terms can be replaced by $1 / 2$, and the integral is then bounded by the one on $\mathbb{R}$. Thus

$$
(\underline{5.42}) \leq \operatorname{const} Q(\rho) \frac{1}{\sqrt{\gamma T^{1 / 3}}} .
$$

In the worse case, when $\gamma \rightarrow 0$, which happens when $\rho \rightarrow z_{c}$, we have $\gamma_{1} T^{1 / 3} \simeq\left(\xi_{1}+\xi_{2}+4 L\right)^{1 / 2} \geq(2 L)^{1 / 2}$, which dominates $\gamma_{2}$ for $L$ large enough.

Therefore we have shown that

$$
\left|\widetilde{K}_{T}^{\text {conj }}\left(u_{1}, \xi_{1} ; u_{2}, \xi_{2}\right)\right| \leq Q(\rho) \mathcal{O}(1) .
$$

It thus remains to find an bound on $Q(\rho)$. We have, by (5.18),

$$
Q(\rho)=e^{\left[\frac{1}{3} \kappa_{2}\left(-\rho-z_{c}\right)^{3}-\left(\tilde{\xi}_{1}+\tilde{\xi}_{2}\right) \frac{1+\sqrt{q}}{\sqrt{q}}\left(-\rho-z_{c}\right) T+\frac{\kappa_{1}}{q}\left(u_{2}-u_{1}\right)\left(-\rho-z_{c}\right)^{2} T^{2 / 3}\right]\left(1+\mathcal{O}\left(-\rho-z_{c}\right)\right)} .
$$

In the case $\left|\tilde{\xi}_{1}+\tilde{\xi}_{2}\right| \leq \varepsilon$, we then obtain

$$
Q(\rho) \leq e^{\left(\xi_{1}+\xi_{2}+4 L\right)^{3 / 2}\left(\frac{1}{3}-\frac{1+\sqrt{q}}{\sqrt{q}}\right) \kappa_{2}^{-1 / 2}(1+\mathcal{O}(\varepsilon))+\left(\xi_{1}+\xi_{2}+4 L\right) \mathcal{O}(1)} \leq \text { const } e^{-\left(\xi_{1}+\xi_{2}\right)}
$$

for $L \gg 1, \varepsilon \ll 1$. Finally, when $\left|\tilde{\xi}_{1}+\tilde{\xi}_{2}\right| \geq \varepsilon$, we have

$$
Q(\rho) \leq e^{-\left(\xi_{1}+\xi_{2}+4 L\right)\left(\left(\frac{1}{3}-\frac{1+\sqrt{q}}{\sqrt{q}}\right) \varepsilon^{1 / 2} T^{1 / 3} \kappa_{2}^{-1 / 2}+\mathcal{O}(1)\right)} \leq e^{-\left(\xi_{1}+\xi_{2}\right)}
$$

by first choosing $\varepsilon>0$ small and then $T$ large enough.

Proof of Theorem 3. The proof of Theorem 3 is the complete analogue of Theorem 2.5 in [5]. The results in Propositions 5.1,5.3,5.4, and 5.5 in [5] are replaced by the ones in Proposition 15, 16, and 17, The strategy is to write the Fredholm series of the expression for finite $T$ and, by using the bounds in Propositions 16 and 17, see that it is bounded by a $T$-independent and integrable function. Once this is proven, one can exchange the sums/integrals and the $T \rightarrow \infty$ limit by the theorem of dominated convergence. For details, see Theorem 2.5 in [5]. 


\section{Proof of Theorem 5}

In this section we prove Theorem 5, By Theorem 1, the right hand side of (2.16) with $n_{i}=\left\lfloor\left(t_{i}-H_{i}-x_{i}\right) / 2\right\rfloor$ can be written as Fredholm determinant of the kernel

$$
\mathbb{1}\left(X_{i}<x_{i}\right) K\left(\left(n_{i}, t_{i}\right), X_{i} ;\left(n_{j}, t_{j}\right), X_{j}\right) \mathbb{1}\left(X_{j}<x_{j}\right)
$$

with $K$ given in (2.4). By the change of variable $X_{i}=-h_{i}+H_{i}+x_{i}$, one obtains the Fredholm determinant of the kernel

$$
\mathbb{1}\left(h_{i}>H_{i}\right) K\left(\left(n_{i}, t_{i}\right), H_{i}+x_{i}-h_{i} ;\left(n_{j}, t_{j}\right), H_{j}+x_{j}-h_{j}\right) \mathbb{1}\left(h_{j}>H_{j}\right) .
$$

With this preparation, we now go to the proof of Theorem 5 .

Proof of Theorem 5. We have to analyze the kernel (2.4) with entries

$$
n_{i}=\frac{\mathbf{t}_{i}+\mathbf{x}_{i}}{\sqrt{q}}-\frac{H_{i}}{2}, \quad t_{i}=\frac{2 \mathbf{t}_{i}}{\sqrt{q}}, \quad x_{i}=-\frac{2 \mathbf{x}_{i}}{\sqrt{q}}+H_{i}-h_{i}
$$

and take the limit $q \rightarrow 0$ with $h_{i}, H_{i}$ fixed. The scaling of $x_{i}$ might look different from the one in (2.17) but, as we can see below, (6.3) with the last one replaced by $x_{i}=-\frac{2 \mathbf{x}_{i}}{\sqrt{q}}$ gives the same limiting kernel. As $q \rightarrow 0$, the kernel does not have a well defined limit and, as usual, we first have to consider a conjugate kernel. More precisely, we define

$$
K_{q}\left(\left(\mathbf{x}_{1}, \mathbf{t}_{1}\right), h_{1} ;\left(\mathbf{x}_{2}, \mathbf{t}_{2}\right), h_{2}\right)=K\left(\left(n_{1}, t_{1}\right), x_{1} ;\left(n_{2}, t_{2}\right), x_{2}\right) q^{\left(x_{1}-x_{2}\right) / 2} \frac{q^{n_{1}-n_{2}}}{q^{\left(t_{1}-t_{2}\right) / 2}}
$$

What we have to prove is

$$
\lim _{q \rightarrow 0} \operatorname{det}\left(\mathbb{1}-\chi_{H} K_{q} \chi_{H}\right)=\operatorname{det}\left(\mathbb{1}-\chi_{H} K^{\mathrm{PNG}} \chi_{H}\right) .
$$

First we prove the pointwise convergence and then we obtain bounds allowing us to take the limit inside the Fredholm determinant.

Consider the term coming from (2.6). By the change of variable $w=$ $-1+\sqrt{q} z$, we get

$$
\frac{1}{2 \pi \mathrm{i}} \oint_{\Gamma_{0}} \frac{\mathrm{d} z}{z} \frac{(\sqrt{q}+(1-q) z)^{t_{1}-t_{2}}}{z^{x_{1}-x_{2}}}\left(\frac{1-\sqrt{q} z}{z(\sqrt{q}+(1-q) z)}\right)^{n_{1}-n_{2}}
$$

and, by inserting (6.3), one obtains

$$
\begin{aligned}
& \frac{1}{2 \pi \mathrm{i}} \oint_{\Gamma_{0}} \frac{\mathrm{d} z}{z}\left(\frac{(\sqrt{q}+(1-q) z)(1-\sqrt{q} z)}{z}\right)^{\left(\mathbf{t}_{1}-\mathbf{t}_{2}\right) / \sqrt{q}} \\
& \left(\frac{\sqrt{q}+(1-q) z}{(1-\sqrt{q} z) z}\right)^{\left(\mathbf{x}_{1}-\mathbf{x}_{2}\right) / \sqrt{q}}\left(\frac{\sqrt{q}+(1-q) z}{(1-\sqrt{q} z) z}\right)^{\left(H_{1}-H_{2}\right) / 2} \frac{1}{z^{h_{2}-h_{1}}} .
\end{aligned}
$$


Consider $q \leq q_{0}$ for some $q_{0}<1$ fixed. Then, we can fix the path $\Gamma_{0}$ independent of $q$, and the $q \rightarrow 0$ limit is easily obtained. It results in

$$
\begin{aligned}
& \lim _{q \rightarrow 0}(\underline{6.7})=\frac{1}{2 \pi \mathrm{i}} \oint_{\Gamma_{0}} \frac{\mathrm{d} z}{z} \frac{1}{z^{h_{2}-h_{1}}} e^{-\left(\mathbf{t}_{1}-\mathbf{t}_{2}\right)\left(z-z^{-1}\right)} e^{\left(\mathbf{x}_{2}-\mathbf{x}_{1}\right)\left(z+z^{-1}\right)} \\
= & \left(\frac{\mathbf{x}_{2}-\mathbf{x}_{1}+\mathbf{t}_{1}-\mathbf{t}_{2}}{\mathbf{x}_{2}-\mathbf{x}_{1}-\mathbf{t}_{1}+\mathbf{t}_{2}}\right)^{\left(h_{1}-h_{2}\right) / 2} I_{h_{1}-h_{2}}\left(2 \sqrt{\left(\mathbf{x}_{2}-\mathbf{x}_{1}\right)^{2}-\left(\mathbf{t}_{1}-\mathbf{t}_{2}\right)^{2}}\right),
\end{aligned}
$$

where we applied (A.4).

It is the turn of the term coming from (2.5). We do the change of variable $z=-w /(w+\sqrt{q})$ and then we insert (6.3). The result is

$$
\begin{aligned}
& \frac{1}{2 \pi \mathrm{i}} \oint_{\Gamma_{0}} \frac{\mathrm{d} w}{w(1+\sqrt{q} w)}\left(\frac{\sqrt{q}+w}{w(1+\sqrt{q} w)}\right)^{\left(\mathbf{t}_{1}+\mathbf{t}_{2}\right) / \sqrt{q}}\left(\frac{w}{1+\sqrt{q} w}\right)^{h_{1}}(w+\sqrt{q})^{h_{2}} \\
& \left(\frac{w}{(w+\sqrt{q})(1+\sqrt{q} w)}\right)^{\left(\mathbf{x}_{1}-\mathbf{x}_{2}\right) / \sqrt{q}+\left(H_{2}-H_{1}\right) / 2}
\end{aligned}
$$

If $\mathbf{x}_{2}-\mathbf{x}_{1}>\mathbf{t}_{1}+\mathbf{t}_{2}$, then for $q$ small enough, the result is identically equal to zero, because the pole at $w=0$. If $\mathbf{x}_{1}-\mathbf{x}_{2}>\mathbf{t}_{1}+\mathbf{t}_{2}$, then the result is also zero, because the residues at all other poles, $\sqrt{q}, 1 / \sqrt{q}$, and $\infty$ vanishes. In the other case, when $\left|\mathbf{x}_{2}-\mathbf{x}_{1}\right|<\mathbf{t}_{1}+\mathbf{t}_{2}$, the apparent pole at $w=-\sqrt{q}$ is actually not there. So, we can choose a $\Gamma_{0}$ independent of $q \leq q_{0}$ for some $q_{0}<1$. Then, we can simply take the limit $q \rightarrow 0$ of the integrand, which leads to

$$
\begin{aligned}
& \lim _{q \rightarrow 0}\left([6.9)=\frac{1}{2 \pi \mathrm{i}} \oint_{\Gamma_{0}} \frac{\mathrm{d} w}{w} w^{h_{1}+h_{2}} e^{\left(\mathbf{t}_{1}+\mathbf{t}_{2}\right)\left(w^{-1}-w\right)} e^{\left(\mathbf{x}_{2}-\mathbf{x}_{1}\right)\left(w+w^{-1}\right)}\right. \\
= & \left(\frac{\mathbf{t}_{1}+\mathbf{t}_{2}+\mathbf{x}_{2}-\mathbf{x}_{1}}{\mathbf{t}_{1}+\mathbf{t}_{2}-\mathbf{x}_{2}+\mathbf{x}_{1}}\right)^{\left(h_{1}+h_{2}\right) / 2} J_{h_{1}+h_{2}}\left(2 \sqrt{\left(\mathbf{t}_{1}+\mathbf{t}_{2}\right)^{2}-\left(\mathbf{x}_{2}-\mathbf{x}_{1}\right)^{2}}\right),
\end{aligned}
$$

where in the last step we made the change of variable $w \rightarrow 1 / w$ and applied (A.3).

To have convergence of the Fredholm determinants we still need some bounds for large values of $h_{1}, h_{2}$. For $q$ small enough, say $q \in\left[0, q_{0}\right]$ for some $q_{0}<1$, we can set in (6.7) $\Gamma_{0}=\{z,|z|=\mathrm{e}\}$ in the case $h_{2} \geq h_{1}$, and $\Gamma_{0}=\left\{z,|z|=\mathrm{e}^{-1}\right\}$ in the case $h_{2}<h_{1}$. Then, we get the bound

$$
|(\underline{6.7})| \leq C_{1} e^{-\left|h_{2}-h_{1}\right|}
$$

for some finite constant $C_{1}$ independent of $q$. Moreover, in (6.9) we can choose $\Gamma_{0}=\left\{z,|z|=\mathrm{e}^{-2}\right\}$, which leads to the bound

$$
|(\underline{6.9})| \leq C_{2} e^{-\left(h_{2}+h_{1}\right)}
$$


with $C_{2}<\infty$ independent of $q \leq q_{0}$. These two bounds are enough to have convergence of the Fredholm determinants. The strategy is exactly the same as in the proof of Theorem 3 .

\section{Proof of Theorem 6}

We analyze the kernel (2.23) with the scalings

$$
\begin{aligned}
\mathbf{x}_{i} & =u_{i} T^{2 / 3}, \\
\mathbf{t}_{i} & =\gamma(0) T+\gamma^{\prime}(0) u_{i} T^{2 / 3}+\frac{\gamma^{\prime \prime}(0)}{2} u_{i}^{2} T^{1 / 3}, \\
h_{i} & =2 \mathbf{t}_{i}+\xi_{i} T^{1 / 3}
\end{aligned}
$$

(See (2.24) and (2.25)). The strategy of he proof is the same as that for Theorem 3 and hence we only give the main differences.

First we consider the first term in (2.23). From (6.8) it is rewritten in the from (5.6) with $g_{0}(z), g_{1}(z)$ replaced by

$$
\begin{aligned}
& g_{0}(z)=\left(u_{2}-u_{1}\right)\left(\gamma^{\prime}(0)(z-1 / z-2 \ln z)+(z+1 / z)\right), \\
& g_{1}(z)=\frac{\gamma^{\prime \prime}(0)}{2}\left(u_{2}^{2}-u_{1}^{2}\right)(z-1 / z-2 \ln z)-\left(\xi_{2}-\xi_{1}\right) \ln z .
\end{aligned}
$$

The critical point of $g_{0}(z)$ is now $z_{c}=1$. The series expansions around $z=z_{c}$ are

$$
\begin{aligned}
& g_{0}(z)=g_{0}\left(z_{c}\right)+\left(u_{2}-u_{1}\right)\left(z-z_{c}\right)^{2}+O\left(\left(z-z_{c}\right)^{3}\right), \\
& g_{1}(z)=g_{1}\left(z_{c}\right)-\left(\xi_{2}-\xi_{1}\right)\left(z-z_{c}\right)+O\left(\left(z-z_{c}\right)^{2}\right) .
\end{aligned}
$$

The steep descent path can be taken to be $\Gamma_{0}=\left\{e^{i \phi}, \phi \in[-\pi, \pi)\right\}$. Then the same arguments as in the proof of Theorem 3 give the first term in (2.7).

Next we consider the second term in (2.23). From (6.10) it is rewritten in the form (5.15) with $f_{0}(z), f_{1}(z), f_{2}(z)$ replaced by

$$
\begin{aligned}
& f_{0}(w)=2 \gamma(0)(1 / w-w+2 \ln w), \\
& f_{1}(w)=\gamma^{\prime}(0)\left(u_{1}+u_{2}\right)(1 / w-w+2 \ln w)+\left(u_{2}-u_{1}\right)(w+1 / w), \\
& f_{2}(w)=\frac{\gamma^{\prime \prime}(0)}{2}\left(u_{1}^{2}+u_{2}^{2}\right)(1 / w-w+2 \ln w)+\left(\xi_{1}+\xi_{2}\right) \ln w .
\end{aligned}
$$

Their series expansions around $z_{c}$ are

$$
\begin{aligned}
& f_{0}(w)=-\frac{2 \gamma(0)}{3}(w-1)^{3}+O\left((w-1)^{4}\right) \\
& f_{1}(w)=f_{1}\left(z_{c}\right)+\left(u_{2}-u_{1}\right)(w-1)^{2}+O\left((w-1)^{3}\right) \\
& f_{2}(w)=f_{2}\left(z_{c}\right)+\left(\xi_{1}+\xi_{2}\right)(w-1)+O\left((w-1)^{2}\right) .
\end{aligned}
$$


The steepest descent path is taken to be $\Gamma_{0}=\left\{\rho e^{i \phi}, \phi \in[-\pi, \pi)\right\}$ with $0<\rho<1$. Using these one obtains the second term in (2.7).

The bounds for the diffusion terms and the main term of the kernel are also proved in the same way as those of Propositions 16 and 17.

\section{A Some integral representations}

In this appendix we list some integral representations of the Bessel functions and the modified Bessel functions (we use the conventions of [1]).

$$
\begin{gathered}
J_{n}(2 t)=\frac{1}{2 \pi \mathrm{i}} \oint_{\Gamma_{0}} \frac{\mathrm{d} z}{z} \frac{e^{t\left(z-z^{-1}\right)}}{z^{n}}, \\
I_{n}(2 t)=\frac{1}{2 \pi \mathrm{i}} \oint_{\Gamma_{0}} \frac{\mathrm{d} z}{z} \frac{e^{t\left(z+z^{-1}\right)}}{z^{n}}, \\
\frac{1}{2 \pi \mathrm{i}} \oint_{\Gamma_{0}} \frac{\mathrm{d} z}{z} \frac{e^{b\left(z-z^{-1}\right)} e^{a\left(z+z^{-1}\right)}}{z^{n}}=\left(\frac{b+a}{b-a}\right)^{n / 2} J_{n}\left(2 \sqrt{b^{2}-a^{2}}\right), \\
\frac{1}{2 \pi \mathrm{i}} \oint_{\Gamma_{0}} \frac{\mathrm{d} z}{z} \frac{e^{b\left(z-z^{-1}\right)} e^{a\left(z+z^{-1}\right)}}{z^{n}}=\left(\frac{a+b}{a-b}\right)^{n / 2} I_{n}\left(2 \sqrt{a^{2}-b^{2}}\right), \\
\frac{-1}{2 \pi \mathrm{i}} \int_{\gamma_{\infty}}^{\mathrm{d} v e^{v^{3} / 3+a v^{2}+b v}}=\operatorname{Ai}\left(a^{2}-b\right) \exp \left(2 a^{3} / 3-a b\right),
\end{gathered}
$$

where $\gamma_{\infty}$ is any path from $e^{\mathrm{i} \pi / 3} \infty$ to $e^{-\mathrm{i} \pi / 3} \infty$.

\section{References}

[1] M. Abramowitz and I.A. Stegun, Pocketbook of mathematical functions, Verlag Harri Deutsch, Thun-Frankfurt am Main, 1984.

[2] J. Baik and E.M. Rains, Symmetrized random permutations, Random Matrix Models and Their Applications, vol. 40, Cambridge University Press, 2001, pp. 1-19.

[3] A.L. Barabási and H.E. Stanley, Fractal concepts in surface growth, Cambridge University Press, Cambridge, 1995. 
[4] A. Borodin and P.L. Ferrari, Large time asymptotics of growth models on space-like paths I: PushASEP, preprint: arXiv:0707.2813 (2007).

[5] A. Borodin, P.L. Ferrari, and M. Prähofer, Fluctuations in the discrete TASEP with periodic initial configurations and the Airy $y_{1}$ process, Int. Math. Res. Papers 2007 (2007), rpm002.

[6] A. Borodin, P.L. Ferrari, M. Prähofer, and T. Sasamoto, Fluctuation properties of the TASEP with periodic initial configuration, preprint: arXiv:math-ph/0608056; To appear in J. Stat. Phys. (2007).

[7] A. Borodin, P.L. Ferrari, and T. Sasamoto, Transition between Airy $_{1}$ and Airy $_{2}$ processes and TASEP fluctuations, preprint: arXiv:math-ph/0703023; To appear in CPAM (2007).

[8] A. Borodin and G. Olshanski, Stochastic dynamics related to Plancherel measure, AMS Transl.: Representation Theory, Dynamical Systems, and Asymptotic Combinatorics (V. Kaimanovich and A. Lodkin, eds.), 2006, pp. 9-22.

[9] P.L. Ferrari, Java animation of the PNG dynamics, http://www.wias-berlin.de/people/ferrari/ homepage/animations/RSKFinal.html.

[10] P.L. Ferrari, Polynuclear growth on a flat substrate and edge scaling of GOE eigenvalues, Comm. Math. Phys. 252 (2004), 77-109.

[11] P.L. Ferrari and M. Prähofer, One-dimensional stochastic growth and Gaussian ensembles of random matrices, Markov Processes Relat. Fields 12 (2006), 203-234.

[12] D.J. Gates and M. Westcott, Stationary states of crystal growth in three dimensions, J. Stat. Phys. 88 (1995), 999-1012.

[13] T. Imamura and T. Sasamoto, Fluctuations of the one-dimensional polynuclear growth model with external sources, Nucl. Phys. B 699 (2004), 487-502.

[14] T. Imamura and T. Sasamoto, Polynuclear growth model with external source and random matrix model with deterministic source, Phys. Rev. E 71 (2005), 041606.

[15] W. Jockush, J. Propp, and P. Shor, Random domino tilings and the arctic circle theorem, arXiv:math.CO/9801068 (1995). 
[16] K. Johansson, Non-intersecting paths, random tilings and random matrices, Probab. Theory Related Fields 123 (2002), 225-280.

[17] K. Johansson, Discrete polynuclear growth and determinantal processes, Comm. Math. Phys. 242 (2003), 277-329.

[18] K. Johansson, The arctic circle boundary and the Airy process, Ann. Probab. 33 (2005), 1-30.

[19] K. Kardar, G. Parisi, and Y.Z. Zhang, Dynamic scaling of growing interfaces, Phys. Rev. Lett. 56 (1986), 889-892.

[20] A. M. Povolotsky and V. B. Priezzhev, Determinant solution for the totally asymmetric exclusion process with parallel update, J. Stat. Mech. (2006), P07002.

[21] M. Prähofer and H. Spohn, Statistical self-similarity of one-dimensional growth processes, Physica A 279 (2000), 342-352.

[22] M. Prähofer and H. Spohn, Universal distributions for growth processes in $1+1$ dimensions and random matrices, Phys. Rev. Lett. 84 (2000), $4882-4885$.

[23] M. Prähofer and H. Spohn, Scale invariance of the PNG droplet and the Airy process, J. Stat. Phys. 108 (2002), 1071-1106.

[24] T. Sasamoto, Spatial correlations of the 1D KPZ surface on a flat substrate, J. Phys. A 38 (2005), L549-L556.

[25] T. Sasamoto, Fluctuations of the one-dimensional asymmetric exclusion process using random matrix techniques, J. Stat. Mech. (2007), P07007.

[26] T. Sasamoto and T. Imamura, Fluctuations of a one-dimensional polynuclear growth model in a half space, J. Stat. Phys. 115 (2004), 749-803.

[27] C.A. Tracy and H. Widom, On orthogonal and symplectic matrix ensembles, Comm. Math. Phys. 177 (1996), 727-754. 